\title{
SELF-CONSISTENT EFFECTIVE EQUATIONS MODELING BLOOD FLOW IN MEDIUM-TO-LARGE COMPLIANT ARTERIES
}

\author{
SUNČICA ČANIĆ* ${ }^{*}$ DANIELE LAMPONI ${ }^{\dagger}$, ANDRO MIKELIĆ ${ }^{\ddagger}$, AND JOSIP TAMBAČA ${ }^{\S}$
}

\begin{abstract}
We study the flow of an incompressible viscous fluid through a long tube with compliant walls. The flow is governed by a given time dependent pressure head difference. The Navier-Stokes equations for an incompressible viscous fluid are used to model the flow, and the Navier equations for a curved, linearly elastic membrane to model the wall. Employing the asymptotic techniques typically used in thin domains, we derive a set of effective equations that hold in medium-to-large compliant vessels for laminar flow regimes. The main novelty is the derivation of the effective equations that do not assume any ad hoc closure, typically assumed in the derivation of one-dimensional models. Using ideas from homogenization theory for porous media flows, we obtain a closed system of effective equations that are of Biot type with memory. Memory accounts for the wave-like phenomena in the problem. Although the equations are two-dimensional, their simple structure enables a design of a numerical algorithm that has complexity of a one-dimensional solver. Our numerical simulations show that our model captures two-dimensional effects that cannot be captured using standard one-dimensional methods.
\end{abstract}

Key words. Blood flow, compliant arteries, fluid-structure interaction, effective equations.

AMS subject classifications. 35Q30, 74K15, 76D27

1. Introduction. In this paper we derive the effective equations that describe the flow of a viscous, incompressible Newtonian fluid in a long elastic tube. The paper is motivated by the study of blood flow in compliant arteries. Although blood is not a Newtonian fluid (it is a suspension of red blood cells, white blood cells and platelets in plasma), the Newtonian assumption is considered acceptable as a first approximation for the flow in medium-to-large vessels, see e.g., [23, 29, 38]. To model arterial walls we employ the Navier equations for a linearly elastic membrane, as suggested in [12,19, 20,23,38], with zero base pressure loading and prestretch in the axial direction. They describe "effective" response of arterial walls, consisting of three layers (intima, media and adventitia), to the forces induced by the pulsatile blood flow. Although the blood vessel walls behave nonlinearly (nonlinear viscoelasticity) linear elasticity has been widely used as a first approximation for the vessel wall behavior.

In spite of all the simplifying assumptions made so far, the mathematical and numerical study of the underlying fluid-structure interaction is a difficult one. Various numerical methods have been successfully proposed to study fluid-structure interactions arising in cardiovascular problems, see e.g. $[13,14,26,30,31,33,38]$. Nevertheless they are still rather involving and time-consuming whenever larger threedimensional sections of the cardiovascular system are simulated. The primary reason lies in the relatively large wall deformations (the diameter of an artery in a healthy human varies up to $10 \%$ of the unstressed configuration). Another difficulty comes

*Department of Mathematics, University of Houston, 4800 Calhoun Rd., Houston TX 772043476, United States (canic@math.uh.edu). This research was supported by the Texas Higher Education Board, ARP grant \#003652-0112-2001 and by the National Science Foundation under grants DMS9970310 and DMS0245513

${ }^{\dagger}$ Institut d'analyse et Calcul Scientifique, Ecole Polytechnique Fédérale de Lausanne, Switzerland (daniele.lamponi@epfl.ch).

${ }^{\ddagger}$ LaPCS, UFR Mathématiques, Université Claude Bernard Lyon 1, 21, bd. Claude Bernard, 69622 Villeurbanne Cedex, France (mikelic@univ-lyon1.fr).

$\S$ Department of Mathematics, University of Zagreb, Bijenička 30, 10000 Zagreb, Croatia (tambaca@math.hr). 
from the fact that the density of the fluid is close to that of the interface, giving rise to the "fully" coupled dynamics. This is why simplified, effective models are called for. In this vein, this paper addresses the derivation of a self-consistent, effective system of equations describing the flow of an axially symmetric, Newtonian fluid through a linearly elastic tube with aspect ratio $\varepsilon=R / L(R=$ radius, $L=$ length of the tube). Using rigorous mathematical approach typical for problems in thin domains, see e.g. [8], we derive the energy and the a priori solution estimates that provide the information about the size of the vessel wall displacement and flow regime, in terms of the parameters of the problem (Young's modulus of the vessel wall, inlet and outlet pressure data, vessel wall thickness, e.t.c.). The a priori estimates provide optimal scalings for the coupled fluid-structure interaction problem. They are used in the asymptotic expansions to obtain the effective equations that approximate the original, three-dimensional problem to the $\epsilon^{2}$-order. The coupling between the fluid and the interface is described by requiring the continuity of velocity and the continuity (balance) of contact forces applied by the fluid and by the membrane. They are evaluated at a deformed interface giving rise to a free-boundary problem for the fluid velocity and structure displacement. In our work the $\epsilon^{2}$-approximation of the balance of contact forces implies a linear contact force coupling, consistent with the assumptions of linear elasticity. Coupling though the deformed interface with the linear relationship between the contact forces has been widely used in literature, $[4,26,28,31,38]$. In particular, standard one-dimensional models, see for example $[3,4,10,16,26,28,34,38]$, have all been obtained by averaging across the vessel crosssection of the corresponding free-boundary problem equations. Due to the nonlinear nature of the problem, the averaging technique requires certain closure assumptions, typically in the form af an axial velocity profile. Using the same approach with our asymptotic expansions, we recover the standard one-dimensional models.

The main contribution of this manuscript is the derivation of a closed set of effective equations for which the closure follows from the underlying three-dimensional equations. We introduce a novel approach that leads to a set of closed effective equations. The approach is based on the standard homogenization techniques used in porous media problems [22, 24]. Using this approach we obtain closed effective equations that are of Biot type with memory. Biot type equations have been used in modeling seismic waves and in general, describing waves in deformable, porous media [1]. Memory terms are typical in effective equations describing wave-like phenomena in the underlying physics. In our case they describe the coupling between the waves in the fluid and the elastic structure. We prove that our equations solve the original, 3 -dimensional problem, to the $\varepsilon^{2}$ accuracy. Although our final equations are twodimensional, they can be solved numerically as a coupled pair of one-dimensional problems. We present a numerical algorithm based on the Finite Difference Method (FDM) to solve for the waves in the elastic structure and on the Finite Element Method (FEM) to solve for the fluid velocity. Our numerical simulations show that, although the complexity of the numerical algorithm is that of the one-dimensional solvers, we capture two-dimensional phenomena up to the $\epsilon^{2}$-order that cannot be captured using one-dimensional models. This paper is organized as follows. We start by defining the problem in $\S 2$. Global weak formulation is presented in $\S 3.1$ and energy estimates are derived in $\S 3.2$. Based on the energy estimates we obtain a priori solution estimates in $\S 4$. They define the leading order behavior in asymptotic expansions, discussed in $\S 5$. In $\S 5$ we also derive a set of reduced, two-dimensional free-boundary problem equations. The averaged, one-dimensional equations using 
standard ad hoc closure are derived in $\S 6$. Finally, in $\S 7$ we obtain the self-contained, simplified, effective equations without an ad hoc closure. Numerical simulations are presented in $\S 8$.

2. Statement of the problem . We consider the unsteady axisymmetric flow of a Newtonian incompressible fluid in a thin elastic right cylinder whose radius is small with respect to its length. Define the aspect ratio (the ratio between the radius and the length of the cylinder) to be $\epsilon=R / L$. For each fixed $\epsilon>0$ introduce

$$
\Omega_{\varepsilon}(t)=\left\{x \in \mathbb{R}^{3} ; x=(r \cos \vartheta, r \sin \vartheta, z), r<R+\eta^{\varepsilon}(z, t), 0<z<L\right\} .
$$

Domain $\Omega_{\varepsilon}(t)$ is filled with fluid modeled by the incompressible Navier-Stokes equations. Assuming zero angular velocity, in cylindrical coordinates the Eulerian formulation of the equations in $\Omega_{\varepsilon}(t) \times \mathbb{R}_{+}$reads

$$
\begin{array}{r}
\rho\left\{\frac{\partial v_{r}^{\varepsilon}}{\partial t}+v_{r}^{\varepsilon} \frac{\partial v_{r}^{\varepsilon}}{\partial r}+v_{z}^{\varepsilon} \frac{\partial v_{r}^{\varepsilon}}{\partial z}\right\}-\mu\left(\frac{\partial^{2} v_{r}^{\varepsilon}}{\partial r^{2}}+\frac{\partial^{2} v_{r}^{\varepsilon}}{\partial z^{2}}+\frac{1}{r} \frac{\partial v_{r}^{\varepsilon}}{\partial r}-\frac{v_{r}^{\varepsilon}}{r^{2}}\right)+\frac{\partial p^{\varepsilon}}{\partial r}=0 \\
\rho\left\{\frac{\partial v_{z}^{\varepsilon}}{\partial t}+v_{r}^{\varepsilon} \frac{\partial v_{z}^{\varepsilon}}{\partial r}+v_{z}^{\varepsilon} \frac{\partial v_{z}^{\varepsilon}}{\partial z}\right\}-\mu\left(\frac{\partial^{2} v_{z}^{\varepsilon}}{\partial r^{2}}+\frac{\partial^{2} v_{z}^{\varepsilon}}{\partial z^{2}}+\frac{1}{r} \frac{\partial v_{z}^{\varepsilon}}{\partial r}\right)+\frac{\partial p^{\varepsilon}}{\partial z}=0 \\
\frac{\partial v_{r}^{\varepsilon}}{\partial r}+\frac{\partial v_{z}^{\varepsilon}}{\partial z}+\frac{v_{r}^{\varepsilon}}{r}=0 .
\end{array}
$$

We assume that the lateral wall of the cylinder, $\Sigma_{\varepsilon}(t)=\left\{r=R+\eta^{\varepsilon}(z, t)\right\} \times(0, L)$, is

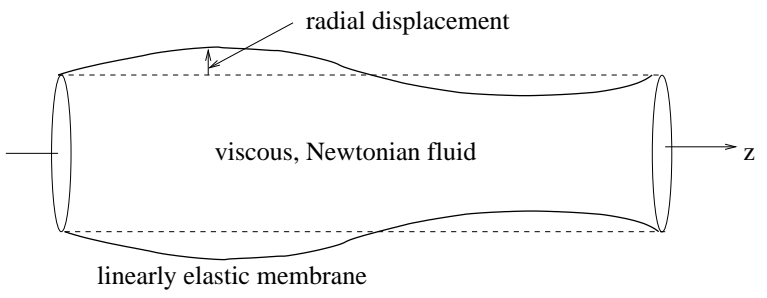

FIG. 2.1. Domain $\Omega_{\varepsilon}(t)$.

elastic and allows only radial displacements. Its motion, described in Lagrangian coordinates, is modeled by the Navier equations for a linearly elastic curved membrane. The radial contact force is given by

$$
F_{r}=-\frac{h(\varepsilon) E(\varepsilon)}{1-\sigma^{2}} \frac{\eta^{\varepsilon}}{R^{2}}+h(\varepsilon) G(\varepsilon) k(\varepsilon) \frac{\partial^{2} \eta^{\varepsilon}}{\partial z^{2}}-\rho_{w} h(\varepsilon) \frac{\partial^{2} \eta^{\varepsilon}}{\partial t^{2}}
$$

where $F_{r}$ is the radial component of external forces (coming from the stresses induced by the fluid), $\eta^{\varepsilon}$ is the radial displacement from the reference state $\Sigma_{\varepsilon}^{0}:=\Sigma_{\varepsilon}(0), h=$ $h(\varepsilon)$ is the membrane thickness, $\rho_{w}$ the wall volumetric mass, $E=E(\varepsilon)$ is the Young's modulus, $0<\sigma \leq 0.5$ the Poisson ratio, $G=G(\varepsilon)$ is the shear modulus and $k=k(\varepsilon)$ is the Timoshenko shear correction factor (see [26, 38, 32]). Typical parameter values for which our analysis holds are presented in Table 2.1. They correspond to the blood flow in iliac arteries (left end of intervals for $R$ and $L$ ) and in the abdominal aorta (right end of the intervals for $R$ and $L$ ), see [28, 27,9].

The fluid equations are coupled with the membrane equation through the lateral boundary conditions requiring continuity of velocity and continuity (balance) of forces. The coupling is evaluated at the deformed interface and in the Lagrangian framework, 


\begin{tabular}{|l|l|}
\hline \hline PARAMETERS & VALUES \\
\hline \hline$\varepsilon$ & $0.02-0.06$ \\
\hline Characteristic radius: $R$ & $0.0025-0.012 \mathrm{~m},[38]$ \\
\hline Characteristic length $: L$ & $0.065-0.2 \mathrm{~m}$ \\
\hline Dynamic viscosity: $\mu$ & $3.5 \times 10^{-3} \mathrm{~kg}(\mathrm{~ms})^{-1}$ \\
\hline Young's modulus: $\mathrm{E}$ & $10^{5}-10^{6} \mathrm{~Pa}=\frac{\mathrm{kg}}{\mathrm{m}^{2}},[23]$ \\
\hline Shear modulus: $\mathrm{G}$ & $E /(2(1+\sigma)),[11,23]$ \\
\hline Timoshenko correction factor $k$ & 1 \\
\hline Wall thickness: $\mathrm{h}$ & $1-2 \times 10^{-3} \mathrm{~m}[38]$ \\
\hline Wall density: $\rho_{w}$ & $1.1 \mathrm{~kg} / \mathrm{m}^{2},[38]$ \\
\hline Blood density: $\rho$ & $1050 \mathrm{~kg} / \mathrm{m}^{3}$ \\
\hline Reference pressure: $P_{0}$ & $13000 \mathrm{~Pa}=97.5 \mathrm{mmHg}$ \\
\hline (Normalized) pressure drop for the aorta & $2.67 \mathrm{~Pa}=0.02 \mathrm{mmHg}[17]$ \\
\hline \hline
\end{tabular}

namely, with respect to the reference configuration $\Sigma_{\varepsilon}^{0}$. More specifically, we require that the fluid velocity evaluated at the deformed interface $\left(R+\eta^{\varepsilon}, z, t\right)$ equals the Lagrangian velocity of the membrane. Recalling that only the radial displacements are non-zero, this reads

$$
\begin{array}{r}
v_{r}^{\varepsilon}\left(R+\eta^{\varepsilon}, z, t\right)=\frac{\partial \eta^{\varepsilon}}{\partial t}(z, t) \quad \text { on } \quad(0, L) \times \mathbb{R}_{+}, \\
v_{z}^{\varepsilon}\left(R+\eta^{\varepsilon}, z, t\right)=0 \quad \text { on }(0, L) \times \mathbb{R}_{+} .
\end{array}
$$

Next we consider balance of forces by requiring that the sum of the radial force given by (2.5) and the radial component of the contact force exerted by the fluid to the membrane is equal to zero. The fluid contact force, typically given in Eulerian coordinates, reads

$$
\left(\left(p^{\varepsilon}-p_{\text {ref }}\right) I-2 \mu D\left(v^{\varepsilon}\right)\right) \vec{n} \vec{e}_{r},
$$

where $D\left(v^{\varepsilon}\right)$ is the rate of strain tensor, i.e., the symmetrized gradient of the velocity

$$
D\left(v^{\varepsilon}\right)=\frac{1}{2}\left(\nabla v^{\varepsilon}+\left(\nabla v^{\varepsilon}\right)^{t}\right)
$$

To perform the coupling in the Lagrangian framework we need the Jacobian of the transformation from Eulerian to Lagrangian coordinates. For this purpose we consider Borel subsets $B$ of $\Sigma_{\varepsilon}^{0}$ and require that

$$
\int_{B}\left(\left(p^{\varepsilon}-p_{\mathrm{ref}}\right) I-2 \mu D\left(v^{\varepsilon}\right)\right) \vec{n} \vec{e}_{r}\left(R+\eta^{\varepsilon}(z, t)\right) \sqrt{1+\left(\frac{\partial \eta^{\varepsilon}}{\partial z}\right)^{2}} d z=\int_{B}-F_{r} R d z
$$

for all $B \subset \Sigma_{\varepsilon}^{0}$, where $J:=\left(1+\left(\frac{\partial \eta^{\varepsilon}}{\partial z}\right)^{2}\right)^{1 / 2}$ is the Jacobian determinant of the mapping transforming $d z$ to $d \Sigma_{\varepsilon}(t) /(2 \pi R)$. Pointwise we get that on $\Sigma_{\varepsilon}^{0} \times \mathbb{R}_{+}$

$$
-F_{r}=\left(\left(p^{\varepsilon}-p_{\text {ref }}\right) I-2 \mu D\left(v^{\varepsilon}\right)\right) \vec{n} \cdot \vec{e}_{r}\left(1+\frac{\eta^{\varepsilon}}{R}\right) \sqrt{1+\left(\frac{\partial \eta^{\varepsilon}}{\partial z}\right)^{2}} .
$$

We will see later in $\S 5.1$ that the $\epsilon^{2}$ approximation of condition (2.9) will imply linear contact force coupling, made precise in the discussion above equation (5.13). Initially, 
the cylinder is filled with fluid and the entire structure is in an equilibrium. The equilibrium state has an initial reference pressure $P_{0}=p_{\text {ref }}$ and the initial velocity zero. We are assuming zero base pressure loading $\Delta P_{0}=0$, although a slight modification of model $(2.5)$, see $[20,21]$, could be used to account for its non zero value. The initial pre-stress of the arterial wall at the equilibrium state is taken into account by the term $k G \frac{\partial^{2} \eta^{\epsilon}}{\partial z^{2}}$. In that case the coefficient in front of the second order derivative represents the longitudinal tension at rest, see $[19,26]$. More typically this term accounts for shear deformations, see for example [32]. A typical choice for $k$ is $k=1$ and in linear elasticity $G=E /\left(2\left(1-\sigma^{2}\right)\right)$. In this work we will be assuming that the coefficient $k G$ is such that $G k\left(1-\sigma^{2}\right) / E$ is of order one or smaller. This is used in equation (5.12) where it was shown that the term with the second order derivative is negligible since it appears at the $\epsilon^{2}$-order. The initial data are given by

$$
\eta^{\varepsilon}=\frac{\partial \eta^{\varepsilon}}{\partial t}=0 \quad \text { and } \quad v^{\varepsilon}=0 \quad \text { on } \Sigma_{\varepsilon}(0) \times\{0\}
$$

A time-dependent pressure head data at the inlet and at the outlet boundary drive the problem. We also assume that the end-points of the tube are fixed, namely that the radial component of the velocity and the radial displacement are equal to zero. Therefore, we have the following inlet and outlet boundary data

$$
\begin{array}{r}
v_{r}^{\varepsilon}=0, p^{\varepsilon}+\rho\left(v_{z}^{\varepsilon}\right)^{2} / 2=P_{1}(t)+p_{\text {ref }} \quad \text { on } \quad\left(\partial \Omega_{\varepsilon}(t) \cap\{z=0\}\right) \times \mathbb{R}_{+}, \\
v_{r}^{\varepsilon}=0, p^{\varepsilon}+\rho\left(v_{z}^{\varepsilon}\right)^{2} / 2=P_{2}(t)+p_{\text {ref }} \quad \text { on } \quad\left(\partial \Omega_{\varepsilon} \cap\{z=L\}\right) \times \mathbb{R}_{+}, \\
\eta^{\varepsilon}=0 \text { for } z=0, \quad \eta^{\varepsilon}=0 \text { for } z=L \text { and } \forall t \in \mathbb{R}_{+} .
\end{array}
$$

We will assume that the pressure drop $A(t)=P_{1}(t)-P_{2}(t) \in C_{0}^{\infty}(0,+\infty)$. Note that physically (physiologically) one should expect non-zero displacements at the end points of the tube (vessel). Fixed outlet boundary typically gives rise to the formation of a boundary layer in the reduced set of equations, see $[5,6]$. In Refs. $[5,6]$ we constructed the boundary layer and showed that it contaminates the flow only in a small neighborhood near the boundary (the boundary layer decays exponentially away from the fixed-end boundary). Although in $[5,6]$ we studied the Stokes problem we expect similar results to hold for the Navier-Stokes equations. It has also been our experience that periodic boundary conditions, although natural in rigid-wall geometries, do not give rise to well-posed limiting (reduced) problems when compliant walls are considered. Therefore, when studying effective, reduced equations for initial-boundary value problems in compliant vessels it is important to take two considerations into account. One is the requirement that the full 3-D axially symmetric problem is well-posed, and the other is that the reduced, effective, 1-D problem be well-posed. These were the primary reasons behind conditions (2.11), (2.12) and (2.13). Therefore, in this paper we study the following initial-boundary-value problem for a coupled fluid-structure interaction driven by a time-dependent pressure head: Problem $\mathrm{P}^{\varepsilon}$ :

For each fixed $\varepsilon>0$, find a solution to (2.2), (2.3) and (2.4) in domain $\Omega_{\varepsilon}(t)$ defined by $(2.1)$, with an elastic lateral boundary $\Sigma_{\varepsilon}(t)$. The lateral boundary conditions are given by the continuity of the velocity (2.6) and (2.7), and by the continuity of radial forces (2.5), where the left hand-side of (2.5) is substituted by (2.9). The boundary conditions at the inlet and outlet boundaries are (2.11) and (2.12) and the behavior of the elastic wall there is prescribed by (2.13). The initial data is given by (2.10). We note that in the rest of the paper we will be using several different terms to describe the vessel wall: tube wall, elastic wall, membrane and structure. They should all be assumed equivalent in this manuscript. 


\section{Weak formulation and energy estimates.}

3.1. Global Weak Formulation. We consider global weak formulation of the coupled problem between the fluid and the structure. In contrast with the approach proposed by Quarteroni and Nobile in [26] where weak formulation is designed for the use of the implicit, fully coupled Arbitrary Lagrangian Eulerian (ALE) algorithms, we present here a weak formulation that is based on a fixed-point approach and a priori solution estimates, suitable for the existence proof of a solution to the nonlinear, coupled problem. The main difficulties in defining a weak formulation stem from the following two facts:

1. Coupling through the deformed interface. The domain geometry is timedependent. More precisely the position of the lateral boundary (in Lagrangian coordinates) is determined by its interaction with the fluid (in Eulerian formulation), and

\section{The fluid equations are nonlinear.}

We deal with the first difficulty by deriving the a priori estimates that provide a bound on the radial displacement which determines the domain size at every time step. The a priori solution estimates are obtained in terms of the elasticity constants that describe the properties of the vessel wall, and the inlet and the outlet pressure that drive the problem. Once we have found the information about the maximum size of the domain, we introduce a fixed, "fictitious" domain of a larger radius, and consider the space of velocity functions defined on the entire fictitious domain, satisfying the a priori bounds that ensure the required size of the radial displacement. We define a solution set to consist of all such velocities and of the interfaces that satisfy the "continuity of velocity" condition at the interface. Among all such candidates we look for the functions that satisfy the integral form of the fluid equations with a lateral boundary condition describing continuity of forces. This is where the second difficulty arises. To deal with the nonlinearity of the equations and with the coupling through the deformed interface at the same time, we introduce a linearization that does not change the energy of the original problem, and then define a solution to the nonlinear problem as a fixed-point of the associated nonlinear mapping. We start by introducing the norms that will be used to measure the size of the inlet and the outlet boundary data. Recall that the inlet and the outlet pressure head data (in fact, the deviation from the reference pressure) are denoted by $P_{1}(t)$ and $P_{2}(t)$, respectively, and that the pressure head difference $P_{2}(t)-P_{1}(t)$ is denoted by $A(t)$. Define

$$
\begin{gathered}
\left\|P_{12}(q, T)\right\|_{\mathcal{V}}^{2}=\max \left\{\left\|P_{1}^{2}\right\|_{\infty},\left\|P_{2}^{2}\right\|_{\infty}\right\}+4 q^{2} T^{2} \frac{1}{T} \int_{0}^{T} \max \left\{{P^{\prime}}_{1}^{2}(q \tau),{P^{\prime}}_{2}^{2}(q \tau)\right\} d \tau \\
\|A(q, T)\|_{\text {aver }}^{2}=\frac{1}{T} \int_{0}^{T}|A(q \tau)|^{2} d \tau \\
\mathcal{P}^{2} \equiv\left\|P_{12}(q, T)\right\|_{\mathcal{V}}^{2}+24 \pi^{2} T^{2}\|A(q, T)\|_{\text {aver }}^{2}
\end{gathered}
$$

where $q$ is the frequency of oscillations. For the pressure data corresponding to iliac arteries, shown in Figure 8.1 at the bottom right plot, a rough value of $\mathcal{P}$ is around $36000 \mathrm{~Pa}$. Motivated by the a priori estimates introduced in $\S 4.2$ we consider the radial 
displacements $\eta^{\varepsilon}$ and the velocities $v^{\varepsilon}$ such that

$$
\begin{gathered}
\sup _{0 \leq t \leq T}\left\{\frac{h(\varepsilon) E(\varepsilon)}{R\left(1-\sigma^{2}\right)}\left\|\eta^{\varepsilon}(t)\right\|_{L^{2}(0, L)}^{2}+\frac{h(\varepsilon) \rho_{w} R}{2}\left\|\frac{\partial \eta^{\varepsilon}}{\partial t}(t)\right\|_{L^{2}(0, L)}^{2}+\right. \\
\left.\frac{G(\varepsilon) h(\varepsilon) R}{2}\left\|\frac{\partial \eta^{\varepsilon}}{\partial z}(t)\right\|_{L^{2}(0, L)}^{2}\right\} \leq 2 \frac{R^{3} L\left(1-\sigma^{2}\right)}{h(\varepsilon) E(\varepsilon)} \mathcal{P}^{2} \\
\frac{2 \mu}{\pi} \int_{0}^{T}\left\|D\left(v^{\varepsilon}\right)(\tau)\right\|_{L^{2}\left(\Omega_{\varepsilon}(\tau)\right)}^{2} d \tau+\frac{\rho}{2 \pi} \sup _{0 \leq \tau \leq T}\left\|v^{\varepsilon}(\tau)\right\|_{L^{2}\left(\Omega_{\varepsilon}(\tau)\right)}^{2} \leq 2 \frac{R^{3} L\left(1-\sigma^{2}\right)}{h(\varepsilon) E(\varepsilon)} \mathcal{P}^{2} .
\end{gathered}
$$

REMARK 3.1. In particular, using the estimate

$$
\max _{[0, L]}\left|\eta^{\varepsilon}\right| \leq \sqrt{2}\left\|\frac{\partial \eta^{\varepsilon}}{\partial z}\right\|^{1 / 2}\left\|\eta^{\varepsilon}\right\|^{1 / 2}
$$

we calculate that if

$$
16 L R \mathcal{P}^{2} \leq h(\varepsilon)^{2} \sqrt{G(\varepsilon)}\left(\frac{E(\varepsilon)}{1-\sigma^{2}}\right)^{3 / 2}
$$

then the maximum radial displacement $\eta^{\varepsilon}$ satisfying (3.4) is 50 percent of the nonstressed vessel radius $R$, namely,

$$
\sup _{0 \leq t \leq T}\left\|\eta^{\varepsilon}(t)\right\|_{C[0, L]} \leq \frac{R}{2}
$$

For a range of data presented in Table 2.1 this condition is satisfied implying that the displacement in a healthy artery will always be less than 50 percent of the non-stressed radius. This is a reasonable result since it is expected that the radial displacement in healthy human arteries does not typically exceed 10 percent. We are now ready to introduce the solution spaces $\Gamma$ and $U$ corresponding to the radial displacement and the velocity. Denote by $R_{\max }$ any number greater than or equal to the maximum radius obtained from (3.4) and let $\Omega_{R_{\max }}=\left(0, R_{\max }\right) \times(0, L)$.

Definition 3.2 (Solution SPACES).

- The space $\Gamma$ consists of all the functions

$$
\gamma \in L^{\infty}\left(0, T ; H^{1}(0, L)\right) \cap W^{1, \infty}\left(0, T ; L^{2}(0, L)\right)
$$

such that $\gamma(t, 0)=\gamma(t, L)=0$ and such that the bound (3.4) is satisfied.

- The space $U$ consists of all the functions

$$
u=\left(u_{r}, u_{z}\right) \in L^{2}\left(0, T ; H^{1 / 2-\delta}\left(\Omega_{R_{\max }}\right) \times H^{1}\left(\Omega_{R_{\max }}\right)\right) \cap L^{\infty}\left(0, T ; L^{2}\left(\Omega_{R_{\max }}\right)^{2}\right)
$$

for some $\delta>0$, such that div $u=0$ in $\Omega_{R_{\max }} \times \mathbb{R}_{+}, u_{r}=0$ for $z=0, L$ and the bound (3.5) is satisfied.

We look for a solution among all the functions $\gamma \in \Gamma$ and $u \in U$ that satisfy the continuity of the velocity condition at the interface $\gamma$ and are extended to the rest of the fictitious domain in a manner specified below. More precisely, let

$$
\Omega_{\gamma}(t)=\{(r, z) \mid 0<r<R+\gamma(t, z), \quad z \in(0, L)\}
$$


and $\Sigma_{\gamma}(t)=\{r=R+\gamma(t, z)\} \times(0, L)$.

DEFINITION 3.3. The set of solution candidates $K$ consists of all the functions $(\gamma, u)$, where $u$ are axially symmetric, such that

$$
\begin{aligned}
& K=\left\{(\gamma, u) \in \Gamma \times U \mid u_{r}(r, z, t)=\frac{\partial \gamma}{\partial t}(z, t) \text { for } R+\gamma(t, z) \leq r<R_{\max }\right. \\
& \left.u_{r} \in H^{1}\left(\Omega_{\gamma}(t)\right) \text { and } u_{z}(r, z, t)=0 \text { for } R+\gamma(t, z)<r<R_{\max }\right\} .
\end{aligned}
$$

Remark 3.4. Note that $K$ is bounded but it is not convex. Also note that the trace of $u_{r}$ at $r=R+\gamma(t, z)$ exists at least as an element of $H^{-1 / 2}$ since div $u=0$ and $u_{z}(R+\gamma(t, z), z, t)=0$. To study the integral form of the coupled fluid-interface equations we define the space of test functions.

Definition 3.5 (The TeSt SPACE). Let

$$
\begin{aligned}
V\left(\Omega_{\gamma}(t)\right)= & \left\{\varphi=\varphi_{r} \vec{e}_{r}+\varphi_{z} \vec{e}_{z} \in H^{1}\left(\Omega_{\gamma}(t)\right)^{2} \mid \varphi_{r}(r, 0)=\varphi_{r}(r, L)=0\right. \\
& \left.\varphi_{z}(R+\gamma(z, t), z)=0 \text { and } \operatorname{div} \varphi=0 \text { in } \Omega_{\gamma}(t) \text { a.e. }\right\}
\end{aligned}
$$

The test space is the space $H^{1}\left(0, T ; V\left(\Omega_{\gamma}(t)\right)\right.$.

Recall that for an axially symmetric vector valued function $\psi=\psi_{r} \vec{e}_{r}+\psi_{z} \vec{e}_{z}$ we have

$$
D(\psi)=\left(\begin{array}{ccc}
\frac{\partial \psi_{r}}{\partial r} & 0 & \frac{1}{2}\left(\frac{\partial \psi_{r}}{\partial z}+\frac{\partial \psi_{z}}{\partial r}\right) \\
0 & \frac{\psi_{r}}{r} & 0 \\
\frac{1}{2}\left(\frac{\partial \psi_{r}}{\partial z}+\frac{\partial \psi_{z}}{\partial r}\right) & 0 & \frac{\partial \psi_{z}}{\partial z}
\end{array}\right) .
$$

Define the matrix norm $|\cdot|$ through the scalar product

$$
\Xi: \Psi=T_{r}\left(\Xi \cdot \Psi^{t}\right), \Xi, \Psi \in \mathbb{R}^{9} .
$$

For each $\varepsilon>0$ we study the following evolution problem. For a given $(\gamma, u) \in K$ find $\left(\eta^{\varepsilon}, v_{r}^{\varepsilon}, v_{z}^{\varepsilon}\right) \in K$ such that $\forall \varphi \in H^{1}\left(0, T ; V\left(\Omega_{\gamma}(t)\right)\right)$ we have

$$
\begin{gathered}
2 \mu \int_{\Omega_{\gamma}(t)} D\left(v^{\varepsilon}\right): D(\varphi) r d r d z+\rho \int_{\Omega_{\gamma}(t)}\left\{\frac{\partial v^{\varepsilon}}{\partial t}+(u(t) \nabla) v^{\varepsilon}\right\} \varphi r d r d z \\
+R \int_{0}^{L}\left\{h(\varepsilon) G(\varepsilon) k(\varepsilon) \frac{\partial \eta^{\varepsilon}}{\partial z} \frac{\partial}{\partial z} \varphi_{r}(R+\gamma, z, t)+\frac{h(\varepsilon) E(\varepsilon)}{1-\sigma^{2}} \frac{\eta^{\varepsilon}}{R^{2}} \varphi_{r}(R+\gamma, z, t)\right\} d z \\
+R \rho_{w} h \int_{0}^{L} \frac{\partial^{2} \eta^{\varepsilon}}{\partial t^{2}} \varphi_{r}(R+\gamma(t, z), z, t) d z=-\left.\int_{0}^{R}\left\{P_{2}(q t)-\left.\frac{\rho}{2}\left(u_{z} v_{z}^{\varepsilon}\right)\right|_{z=L}\right\} \varphi_{z}\right|_{z=L} r d r \\
+\left.\int_{0}^{R}\left\{P_{1}(q t)-\left.\frac{\rho}{2}\left(u_{z} v_{z}^{\varepsilon}\right)\right|_{z=0}\right\} \varphi_{z}\right|_{z=0} r d r,
\end{gathered}
$$

and

$$
\eta^{\varepsilon}=\frac{\partial \eta^{\varepsilon}}{\partial t}=0 \quad \text { on } \quad(0, L) \times\{0\} \quad \text { and } v^{\varepsilon}(r, z, 0)=0 .
$$

Scalar products with $\frac{\partial v^{\varepsilon}}{\partial t}$ and $\frac{\partial^{2} \eta^{\varepsilon}}{\partial t^{2}}$ should be understood as duality pairings. Problem (3.10)-(3.11) defines a nonlinear mapping $\Phi$ defined on $K$. The a priori estimates, presented in $\S 4.2$, imply that $\Phi$ maps $K$ to $K$. 
Lemma 3.6. $\Phi(K) \subseteq K$.

Definition 3.7 (Weak SOlution). The triple $\left(\eta^{\varepsilon}, v_{r}^{\varepsilon}, v_{z}^{\varepsilon}\right) \in K$ is a weak solution for the problem $\mathrm{P}^{\varepsilon}$ if it is a fixed point for the mapping $\Phi$.

Existence of a weak solution is a subject of our current research. A related work on the existence of a solution to an incompressible fluid-elastic structure coupled problem can be found in [15]. In the present paper we present the energy estimate and the a priori estimates that determine the "optimal" leading order behavior of the solution in terms of the small parameter $\varepsilon$ which we will use to derive the reduced, effective equations.

3.2. Energy Estimate. The energy of this problem, obtained by using the velocity field as a test function in (3.10), consists of the elastic energy of the membrane, the kinetic and the viscous energy of the fluid, and the energy due to the outside forcing. To get to the energy estimate we start by conveniently rewriting the elastic energy of the membrane, defined by

$$
\mathcal{E}_{e l} \equiv R \int_{0}^{L}\left\{h(\varepsilon) G(\varepsilon) k(\varepsilon) \frac{\partial \eta^{\varepsilon}}{\partial z} \frac{\partial^{2} \eta^{\varepsilon}}{\partial z \partial t}+\frac{h(\varepsilon) E(\varepsilon)}{1-\sigma^{2}} \frac{\eta^{\varepsilon}}{R^{2}} \frac{\partial \eta^{\varepsilon}}{\partial t}+R \rho_{w} h(\varepsilon) \frac{\partial^{2} \eta^{\varepsilon}}{\partial t^{2}} \frac{\partial \eta^{\varepsilon}}{\partial t}\right\} d z
$$

in the following form.

Lemma 3.8. The displacement $\eta^{\varepsilon}$ satisfies

$$
\begin{aligned}
\mathcal{E}_{e l}= & R \frac{d}{2 d t}\left\{\rho_{w} h(\varepsilon) \int_{0}^{L}\left|\frac{\partial \eta^{\varepsilon}}{\partial t}\right|^{2} d z+h(\varepsilon) G(\varepsilon) k(\varepsilon) \int_{0}^{L}\left|\frac{\partial \eta^{\varepsilon}}{\partial z}\right|^{2} d z\right. \\
& \left.+\frac{h(\varepsilon) E(\varepsilon)}{1-\sigma^{2}} \int_{0}^{L}\left|\frac{\eta^{\varepsilon}}{R}\right|^{2}\right\} .
\end{aligned}
$$

This will be used in Proposition 3.9 to obtain the variational equality from which the energy estimate will follow. Next we introduce a time scale in the problem. We are interested in the oscillations of the membrane that are due to the coupled fluidstructure response to the time-dependent pressure (pressure head) drop $A(t)$ and the main pressure head at the inlet and at the outlet boundary, $P_{1}(t)$ and $P_{2}(t)$. These oscillations generally occur at a different time scale than the physical time $t$. The time scale should depend not only on the pressure head data but also on the parameters in the problem. For example, for a stiffer wall, the vibrations of the wall occur at a shorter time scale (high frequency) than the oscillations of a more elastic wall. To capture the waves of the coupled fluid-structure response to the outside forcing we introduce

$$
\tilde{t}=\omega^{\varepsilon} t
$$

where the characteristic frequency $\omega^{\varepsilon}$ will be specified later, see (4.8).

From this point on we use the rescaled time $\tilde{t}$ and drop the symbol wiggle. We 
now derive the variational equality. The following identities will be useful.

$$
\begin{gathered}
\int_{\Omega_{\varepsilon}(t)} \omega^{\varepsilon} \frac{\partial v^{\varepsilon}}{\partial t} v^{\varepsilon} d x=\omega^{\varepsilon} \frac{d}{2 d t} \int_{\Omega_{\varepsilon}(t)}\left|v^{\varepsilon}(t)\right|^{2} d x-\frac{1}{2} \int_{\partial \Omega_{\varepsilon}(t)}\left|v^{\varepsilon}(t)\right|^{2} v^{\varepsilon}(t) \cdot n d S \\
\int_{\Omega_{\varepsilon}(t)}\left(v^{\varepsilon} \cdot \nabla\right) v^{\varepsilon} \cdot v^{\varepsilon} d x=\frac{1}{2} \int_{\partial \Omega_{\varepsilon}(t)}\left|v^{\varepsilon}(t)\right|^{2} v^{\varepsilon}(t) \cdot n d S \\
\int_{\Omega_{\varepsilon}(t)}\left(\nabla p^{\varepsilon}-\mu \Delta v^{\varepsilon}\right) \cdot v^{\varepsilon} d x=\int_{\Omega_{\varepsilon}(t)} \operatorname{Div}\left(p^{\varepsilon} I-2 \mu D\left(v^{\varepsilon}\right)\right) v^{\varepsilon} d x= \\
2 \mu\left\|D\left(v^{\varepsilon}(t)\right)\right\|_{L^{2}\left(\Omega_{\varepsilon}\right)}^{2}+\int_{\partial \Omega_{\varepsilon}(t)}\left(p^{\varepsilon} I-2 \mu D\left(v^{\varepsilon}\right)\right) n v^{\varepsilon} d S
\end{gathered}
$$

Furthermore, using (2.6) in (2.9) we have

$$
\int_{\Sigma_{\varepsilon}(t)}\left(p^{\varepsilon} I-2 \mu D\left(v^{\varepsilon}\right)\right) n v^{\varepsilon} d S=-\int_{0}^{L} F_{r}(t, z) \omega^{\varepsilon} \frac{\partial \eta^{\varepsilon}}{\partial t}(t, z) d z
$$

By keeping the rescaled time in mind, and by using the expression for the elastic energy (3.12) and the above identities we obtain

Proposition 3.9 (Variational Equality). Solution $\left(v_{r}^{\varepsilon}, v_{z}^{\varepsilon}, \eta^{\varepsilon}\right)$ of problem (3.10)-(3.11) satisfies the following variational equality

$$
\begin{aligned}
& \omega^{\varepsilon} h(\varepsilon) \frac{d}{2 d t}\left\{\rho\left(\omega^{\varepsilon}\right)^{2} \rho_{w} R\left\|\frac{\partial \eta^{\varepsilon}(t)}{\partial t}\right\|_{L^{2}(0, L)}^{2}+G(\varepsilon) k(\varepsilon) R\left\|\frac{\partial \eta^{\varepsilon}(t)}{\partial z}\right\|_{L^{2}(0, L)}^{2}\right. \\
& \left.+\frac{E(\varepsilon) R}{1-\sigma^{2}}\left\|\frac{\eta^{\varepsilon}(t)}{R}\right\|_{L^{2}(0, L)}^{2}\right\}+\frac{\rho \omega^{\varepsilon}}{2 \pi} \frac{d}{2 d t}\left\|v^{\varepsilon}(t)\right\|_{L^{2}\left(\Omega_{\varepsilon}(t)\right)}^{2}+\frac{\mu}{\pi}\left\|D\left(v^{\varepsilon}(t)\right)\right\|_{L^{2}\left(\Omega_{\varepsilon}(t)\right)}^{2}= \\
& -\int_{0}^{R} P_{2}(q t) v_{z}^{\varepsilon}(t, r, L) r d r+\int_{0}^{R} P_{1}(q t) v_{z}^{\varepsilon}(t, r, 0) r d r
\end{aligned}
$$

with $v_{r}^{\varepsilon}\left(t, R+\eta^{\varepsilon}, z\right)=\omega^{\varepsilon} \frac{\partial \eta^{\varepsilon}}{\partial t}(t, z)$ and $v_{z}^{\varepsilon}\left(t, R+\eta^{\varepsilon}, z\right)=0$ on $(0, L) \times(0, T)$.

Here $q$ corresponds to the frequency of the time-oscillations of the inlet and of the outlet boundary data. Even thought nothing in the analysis presented in this paper requires time-periodic data, we have introduced an explicit frequency parameter $q$ to suggest that the blood flow application typically exhibits time-periodicity. To get the energy estimate in terms of the data we need to estimate the right hand side of the variational equality. Notice that since the axial component of the velocity at the inlet and at the outlet boundary is not prescribed we need to estimate the right hand-side in terms of the data and the energy of the problem. Notice that on the left hand side we only have the $L^{2}$-norm of $D\left(v^{\varepsilon}\right)$ and not the $L^{2}$-norm of $\nabla v^{\varepsilon}$, and so the standard approach based on using the Gronwall estimate and the $L^{2}$-norm of the velocity, $\rho \int_{\Omega_{\varepsilon}}\left|v^{\varepsilon}(t)\right|^{2} r d r d z$, is insufficient to guarantee the correct order of magnitude of the velocity. To get around this difficulty we transform the right hand side term in (3.18) into a combination of a volume term and a lateral boundary term 
as follows

$$
\begin{gathered}
-\int_{0}^{R} P_{2}(q t) v_{z}^{\varepsilon}(t, r, L) r d r+\int_{0}^{R} P_{1}(q t) v_{z}^{\varepsilon}(t, r, 0) r d r= \\
-\int_{\Omega_{\varepsilon}(t)} \frac{A(q t)}{2 \pi L} v_{z}^{\varepsilon} d x+\int_{\Sigma_{\varepsilon}(t)}\left(A(q t) \frac{z}{L}+P_{1}(q t)\right) v_{r}^{\varepsilon}(t, R, z) \frac{n_{r}}{2 \pi} d \Sigma_{\varepsilon}(t)= \\
-\int_{\Omega_{\varepsilon}(t)} \frac{A(q t)}{2 \pi L} v_{z}^{\varepsilon} d x+R \omega^{\varepsilon} \int_{0}^{L}\left(A(q t) \frac{z}{L}+P_{1}(q t)\right) \frac{\partial \eta^{\varepsilon}(t)}{\partial t}(t, z) d z= \\
-\int_{\Omega_{\varepsilon}(t)} \frac{A(q t)}{2 \pi L} v_{z}^{\varepsilon} d x+R \omega^{\varepsilon} \frac{d}{d t} \int_{0}^{L}\left(A(q t) \frac{z}{L}+P_{1}(q t)\right) \eta^{\varepsilon}(t) d z- \\
R \omega^{\varepsilon} \int_{0}^{L}\left(A^{\prime}(q t) \frac{z}{L}+P_{1}^{\prime}(q t)\right) \eta^{\varepsilon}(t, z) d z
\end{gathered}
$$

where $n_{r}=1 / \sqrt{1+\left|\frac{\partial \eta^{\varepsilon}(t)}{\partial z}\right|^{2}}$ and $n_{r} J=1$. We first estimate the lateral boundary terms from (3.19). The following notation will be useful

$$
\|P(t)\|_{\mathcal{H}}^{2}=\max \left\{P_{1}^{2}(q t), P_{2}^{2}(q t)\right\}+q^{2} \int_{0}^{t} \max \left\{{P^{\prime}}_{1}^{2}(q \tau),{P^{\prime}}_{2}^{2}(q \tau)\right\} d \tau
$$

Lemma 3.10. Let $\alpha>0$. Radial displacement $\eta^{\varepsilon}$ satisfies the following estimate

$$
\begin{aligned}
& R \omega^{\varepsilon}\left(\int_{0}^{L}\left(A(q t) \frac{z}{L}+P_{1}(q t)\right) \eta^{\varepsilon}(t, z) d z-\int_{0}^{t} \int_{0}^{L}\left(A^{\prime}(q \tau) \frac{z}{L}+P^{\prime}{ }_{1}(q \tau)\right) \eta^{\varepsilon}(\tau, z) d z d \tau\right) \\
& \leq \frac{h(\varepsilon) E(\varepsilon) \omega^{\varepsilon}}{4 R\left(1-\sigma^{2}\right)}\left\{\left\|\eta^{\varepsilon}(t)\right\|_{L^{2}(0, L)}^{2}+\alpha \int_{0}^{t}\left\|\eta^{\varepsilon}(\tau)\right\|_{L^{2}(0, L)}^{2} d \tau\right\}+\frac{R^{3} L\left(1-\sigma^{2}\right) \omega^{\varepsilon}}{2 h(\varepsilon) E(\varepsilon)}\|P(t)\|_{\mathcal{H}}^{2} .
\end{aligned}
$$

To estimate the volume forcing term in (3.19) we have two possibilities. The first one is to get an estimate in terms of the viscous energy via a variant of Korn's and Poincaré's inequalities. This approach, however, leads to an estimate in terms of the $L^{4}$-norm of $\eta^{\epsilon}$, which we do not control. The second approach is to estimate the volume term via the inertia term. This will lead to the energy estimate (3.23). More precisely, we have the following.

LEMma 3.11. The following estimate holds

$$
\begin{aligned}
\left|\int_{\Omega_{\varepsilon}(t)} \frac{A(q t)}{2 \pi L} v_{z}^{\varepsilon} r d r d z\right| \leq & \frac{3 R^{2} \pi^{2}|A(q t)|^{2}}{L \rho \omega^{\varepsilon} \alpha}+\frac{\alpha \omega^{\varepsilon} \rho}{4 \pi}\left\|v_{z}^{\varepsilon}(t)\right\|_{L^{2}\left(\Omega_{\varepsilon}\right)}^{2} \\
& +\frac{3 \pi^{2}|A(q t)|^{2}}{\alpha L^{2} \rho \omega^{\varepsilon}}\left\|\eta^{\varepsilon}\right\|_{L^{2}(0, L)}^{2} .
\end{aligned}
$$

Proof. We have

$$
\begin{gathered}
\left|\int_{\Omega_{\varepsilon}(t)} \frac{A(q t)}{2 \pi L} v_{z}^{\varepsilon} r d r d z\right| \leq \frac{|A(q t)|}{L}\left\|v_{z}^{\varepsilon}(t)\right\|_{L^{2}\left(\Omega_{\varepsilon}(t)\right)}\left|\Omega_{\varepsilon}(t)\right|^{1 / 2} \leq \\
\frac{\alpha \omega^{\varepsilon} \rho}{4 \pi}\left\|v_{z}^{\varepsilon}(t)\right\|_{L^{2}\left(\Omega_{\varepsilon}(t)\right)}^{2}+\frac{\pi|A(q t)|^{2}}{\alpha L^{2} \omega^{\varepsilon} \rho}\left|\Omega_{\varepsilon}(t)\right|
\end{gathered}
$$


As $\left|\Omega_{\varepsilon}(t)\right|=\pi \int_{0}^{L}\left(R+\eta^{\varepsilon}\right)^{2} d z$ we get (3.21).

Finally, after integrating (3.18) with respect to time, and using (3.19) and the time-integrated (3.21), we get

Theorem 3.12 (Energy estimate). Radial displacement $\eta^{\varepsilon}$, the displacement gradient $\partial \eta^{\varepsilon} / \partial z$, the kinetic energy of the membrane $\rho_{w}\left\|\frac{\partial \eta^{\varepsilon}}{\partial t}(t)\right\|_{L^{2}(0, L)}^{2}$, the viscous energy $\mu\left\|D\left(v^{\varepsilon}\right)\right\|_{L^{2}\left(\Omega_{\varepsilon}\right)}^{2}$ and the kinetic energy $\rho\left\|v^{\varepsilon}\right\|_{L^{2}\left(\Omega_{\varepsilon}\right)}^{2}$ of the fluid, satisfy the following energy estimate

$$
\begin{gathered}
\omega^{\varepsilon} \frac{h(\varepsilon) E(\varepsilon)}{4 R\left(1-\sigma^{2}\right)}\left\|\eta^{\varepsilon}(t)\right\|_{L^{2}(0, L)}^{2}+\left(\omega^{\varepsilon}\right)^{3} \frac{h(\varepsilon) \rho_{w} R}{2}\left\|\frac{\partial \eta^{\varepsilon}}{\partial t}(t)\right\|_{L^{2}(0, L)}^{2} \\
+\omega^{\varepsilon} \frac{G(\varepsilon) h(\varepsilon) R}{2}\left\|\frac{\partial \eta^{\varepsilon}}{\partial z}(t)\right\|_{L^{2}(0, L)}^{2}+\frac{\mu}{\pi} \int_{0}^{t}\left\|D\left(v^{\varepsilon}\right)(\tau)\right\|_{L^{2}\left(\Omega_{\varepsilon}(\tau)\right)}^{2} d \tau \\
+\frac{\omega^{\varepsilon} \rho}{4 \pi}\left\|v^{\varepsilon}(\tau)\right\|_{L^{2}\left(\Omega_{\varepsilon}(\tau)\right)}^{2} \leq \\
\left\{\omega^{\varepsilon} \alpha \frac{h(\varepsilon) E(\varepsilon)}{4 R\left(1-\sigma^{2}\right)}+\frac{3 \pi^{2}\|A\|_{L^{\infty}(0, t)}^{2}}{\rho L^{2} \omega^{\varepsilon} \alpha}\right\} \int_{0}^{t}\left\|\eta^{\varepsilon}(\tau)\right\|_{L^{2}(0, L)}^{2} d \tau \\
+\frac{\omega^{\varepsilon} \rho \alpha}{4 \pi} \int_{0}^{t}\left\|v^{\varepsilon}(\tau)\right\|_{L^{2}\left(\Omega_{\varepsilon}(\tau)\right)}^{2} d \tau+\frac{R^{3} L\left(1-\sigma^{2}\right) \omega^{\varepsilon}}{2 h(\varepsilon) E(\varepsilon)}\|P(t)\|_{\mathcal{H}}^{2}+\frac{3 R^{2} \pi^{2}}{\alpha \omega^{\varepsilon} \rho L} \int_{0}^{t}|A(q \tau)|^{2} d \tau .
\end{gathered}
$$

We use this energy inequality to obtain the a priori solution estimates.

4. A priori solution estimates. We first focus on the case when the pressure head difference between the inlet and the outlet boundary is zero, $A(t)=0$.

4.1. A priori solution estimates when $A(t)=0$. We are interested in studying a coupled response of the fluid and the structure to a time-dependent pressure head with zero pressure (pressure head) drop. The energy stored in the membrane due to the time-dependent pressure head will impact the movement of the fluid in the tube. Our result presented below shows that the estimates for the radial displacement of the tube and for the velocity of the fluid are independent of the time scale $\omega^{\varepsilon}$. The amplitude of the oscillations as well as the magnitude of the fluid velocity depends on the elasticity properties of the tube walls, as well as on the radius, the length of the tube and the magnitude and frequency of the pressure head.

Lemma 4.1. Let $A(t)=0$. Then the estimates for $\eta^{\varepsilon}$ and $v^{\varepsilon}$ are independent of $\omega^{\varepsilon}$ and they read

$$
\begin{gathered}
\frac{h(\varepsilon) E(\varepsilon)}{R\left(1-\sigma^{2}\right)}\left\|\eta^{\varepsilon}(t)\right\|_{L^{2}(0, L)}^{2}+\frac{\rho}{2 \pi}\left\|v^{\varepsilon}(\tau)\right\|_{L^{2}\left(\Omega_{\varepsilon}(\tau)\right)}^{2} \leq \\
\frac{2 R^{3} L\left(1-\sigma^{2}\right)}{h(\varepsilon) E(\varepsilon)}\left\{\max _{0 \leq t \leq T} P_{1}^{2}(q t)+2 q^{2} T^{2} \frac{1}{T} \int_{0}^{T}\left|P^{\prime}{ }_{1}(q \tau)\right|^{2} d \tau\right\}, \forall t \in[0, T] .
\end{gathered}
$$

Proof. Denote

$$
y(t)=\int_{0}^{t}\left\{\frac{h(\varepsilon) E(\varepsilon)}{R\left(1-\sigma^{2}\right)}\left\|\eta^{\varepsilon}(t)\right\|_{L^{2}(0, L)}^{2}+\frac{\rho}{\pi}\left\|v^{\varepsilon}(\tau)\right\|_{L^{2}\left(\Omega_{\varepsilon}(\tau)\right)}^{2}\right\} d \tau
$$


Suppose that the time oscillations in $P_{1}$ are of order $q$, i.e., that the period of oscillations $T=2 \pi / q$. Then for any $\alpha>0$ we have

$$
y^{\prime}(t) \leq \alpha y(t)+\frac{2 R^{3} L\left(1-\sigma^{2}\right)}{h(\varepsilon) E(\varepsilon)}\left\{\max _{0 \leq t \leq T} P_{1}^{2}(q t)+\frac{q^{2}}{\alpha} \int_{0}^{t}\left|P^{\prime}{ }_{1}(q \tau)\right|^{2} d \tau\right\} ; \quad y(0)=0 .
$$

By applying the Gronwall inequality and by choosing $\alpha=\frac{1}{2 T}$ we get

$$
\begin{gathered}
\frac{h(\varepsilon) E(\varepsilon)}{R\left(1-\sigma^{2}\right)}\left\|\eta^{\varepsilon}(t)\right\|_{L^{2}(0, L)}^{2}+\frac{\rho}{2 \pi}\left\|v^{\varepsilon}(\tau)\right\|_{L^{2}\left(\Omega_{\varepsilon}(\tau)\right)}^{2} \leq \\
\frac{4 R^{3} L\left(1-\sigma^{2}\right)}{h(\varepsilon) E(\varepsilon)}\left\{\max _{0 \leq t \leq T} P_{1}^{2}(q t)+2 q^{2} T \int_{0}^{t}\left|P^{\prime}{ }_{1}(q \tau)\right|^{2} d \tau\right\} \quad \forall t \in[0, T] .
\end{gathered}
$$

4.2. A priori solution estimates for general $A(t)$. In this case we will see that the a priori solution estimates depend on frequency $\omega^{\varepsilon}$. Define

$$
y(t)=\omega^{\varepsilon} \int_{0}^{t}\left\{\frac{h(\varepsilon) E(\varepsilon)}{R\left(1-\sigma^{2}\right)}\left\|\eta^{\varepsilon}(t)\right\|_{L^{2}(0, L)}^{2}+\frac{\rho}{\pi}\left\|v^{\varepsilon}(\tau)\right\|_{L^{2}\left(\Omega_{\varepsilon}(\tau)\right)}^{2}\right\} d \tau .
$$

Then, energy inequality (3.23) implies

$$
\begin{gathered}
y^{\prime}(t) \leq\left(\alpha+\frac{12 \pi^{2}\|A\|_{L^{\infty}(0, t)}^{2} R\left(1-\sigma^{2}\right)}{\rho h(\varepsilon) E(\varepsilon) L^{2}\left(\omega^{\varepsilon}\right)^{2} \alpha}\right) y(t) \\
+\frac{2 R^{3} L\left(1-\sigma^{2}\right) \omega^{\varepsilon}}{h(\varepsilon) E(\varepsilon)}\left\{\max \left\{P_{1}^{2}(q t), P_{2}^{2}(q t)\right\}+\frac{q^{2}}{\alpha} \int_{0}^{t} \max \left\{{P^{\prime}}_{1}^{2}(q \tau),{P_{2}^{\prime 2}}_{2}^{2}(q \tau)\right\} d \tau\right\} \\
+\frac{12 R^{2} \pi^{2}}{\alpha \omega^{\varepsilon} \rho L} \int_{0}^{t}|A(q \tau)|^{2} d \tau ; \quad y(0)=0
\end{gathered}
$$

Without loss of generality suppose

$$
\frac{12 \pi^{2}\|A\|_{L^{\infty}(0, t)}^{2} R\left(1-\sigma^{2}\right)}{\rho h(\varepsilon) E(\varepsilon) L^{2}\left(\omega^{\varepsilon}\right)^{2} \alpha} \leq \alpha
$$

and then choose

$$
\alpha=\frac{1}{4 T} .
$$

Let us note that $\omega^{\varepsilon}$ has dimension $\sec ^{-1}$ and that $\alpha$ and $T$ are dimensionless. Also $T$ is of order one. Let $t_{0} \in[0, T]$ be such that $y^{\prime}\left(t_{0}\right)=\max _{0 \leq t \leq T} y^{\prime}(t)$. Then, instead of using the Gronwall inequality to estimate $y^{\prime}(t)$ we express $y(t)$ on the right hand-side of (4.4) in terms of $y^{\prime}\left(t_{0}\right)$, and use (4.5) to get

$$
\begin{gathered}
y^{\prime}\left(t_{0}\right) \leq 2 \alpha T y^{\prime}\left(t_{0}\right)+\frac{2 R^{3} L\left(1-\sigma^{2}\right) \omega^{\varepsilon}}{h(\varepsilon) E(\varepsilon)}\left\{\max \left\{\left\|P_{1}^{2}\right\|_{\infty},\left\|P_{2}^{2}\right\|_{\infty}\right\}\right. \\
\left.+4 q^{2} T^{2} \frac{1}{T} \int_{0}^{T} \max \left\{P_{1}^{\prime 2}(q \tau), P_{2}^{\prime 2}(q \tau)\right\} d \tau\right\}+\frac{48 R^{2} \pi^{2} T^{2}}{\omega^{\varepsilon} \rho L} \frac{1}{T} \int_{0}^{T}|A(q \tau)|^{2} d \tau ; \\
y(0)=0 .
\end{gathered}
$$


By choosing $\alpha$ as given in (4.6) and by utilizing the notation for the norms defined in (3.1) and (3.2), we get

$$
\frac{1}{2} y^{\prime}\left(t_{0}\right) \leq \frac{2 R^{3} L\left(1-\sigma^{2}\right) \omega^{\varepsilon}}{h(\varepsilon) E(\varepsilon)}\left\|P_{12}(q, T)\right\|_{\mathcal{V}}^{2}+\frac{48 R^{2} \pi^{2} T^{2}}{\omega^{\varepsilon} \rho L}\|A(q, T)\|_{\text {aver }}^{2} .
$$

Now we choose the characteristic time scale, or the characteristic frequency $\omega^{\varepsilon}$, by requiring to see the effects of both the pressure head data, $P_{1}(t)$ and $P_{2}(t)$, as well as the pressure drop data, $A(t)$. More precisely, we choose $\omega^{\varepsilon}$ in (4.7) so that the coefficients on the right hand-side have the same "weight" in $\epsilon$. This leads to

$$
\omega^{\varepsilon}=\frac{2}{L} \sqrt{\frac{2 h(\varepsilon) E(\varepsilon)}{R \rho\left(1-\sigma^{2}\right)}}
$$

Notice that $c=L \omega^{\varepsilon}$ is the characteristic wave speed (the local pulse wave velocity or sound speed). Expression (4.8) leads to the same characteristic wave speed as obtained in equation (16) in Fung's "Biomechanics: Circulation", [11]. For the data presented in Table 2.1 this leads to the pulse wave velocity at the order of $10 \mathrm{~m} / \mathrm{s}$, for the vessel wall having the Young's modulus around $6 \times 10^{5} \mathrm{~Pa}$. This is in good agreement with the measured pulse wave velocity presented in [25]. With this choice of the time-scale the following a priori estimate follows.

Lemma 4.2. The radial displacement $\eta^{\varepsilon}$ and the fluid velocity $v^{\varepsilon}$ satisfy

$$
\frac{h(\varepsilon) E(\varepsilon)}{R\left(1-\sigma^{2}\right)}\left\|\eta^{\varepsilon}(t)\right\|_{L^{2}(0, L)}^{2}+\frac{\rho}{\pi}\left\|v^{\varepsilon}(\tau)\right\|_{L^{2}\left(\Omega_{\varepsilon}(\tau)\right)}^{2} \leq 4 \frac{R^{3} L\left(1-\sigma^{2}\right)}{h(\varepsilon) E(\varepsilon)} \mathcal{P}^{2},
$$

where $\mathcal{P}$ is given by (3.3). Notice that with this choice of $\omega^{\varepsilon}$ inequality (4.5) reads

$$
4 \pi T\left(1-\sigma^{2}\right)\|A\|_{L^{\infty}(0, T)} \leq \frac{h(\varepsilon) E(\varepsilon)}{R}
$$

which holds true for our data since Table 2.1 implies that the left hand side of (4.9) is approximately equal to $10^{-1}$, whereas the right hand side is greater than $10^{4}$. After summarizing those estimates, we get an estimate which is crucial in determining the leading-order behavior in asymptotic expansions. The estimate is a basis for the a priori solution estimates in terms of the small parameter $\epsilon$.

Proposition 4.3 (A PRIORI ESTIMATES WHEN INERTIAL FORCES DOMINATE VISCOUS FORCES). Solution $\left(v_{r}^{\varepsilon}, v_{z}^{\varepsilon}, \eta^{\varepsilon}\right)$ of problem (3.10)-(3.11) satisfies the following a priori estimates

$$
\begin{gathered}
\frac{1}{L}\left\|\eta^{\varepsilon}(t)\right\|_{L^{2}(0, L)}^{2} \leq 4 \frac{R^{4}\left(1-\sigma^{2}\right)^{2}}{h(\varepsilon)^{2} E(\varepsilon)^{2}} \mathcal{P}^{2} \\
\left\|v^{\varepsilon}\right\|_{L^{2}\left(\Omega_{\varepsilon}(t)\right)}^{2} \leq 4 \pi \frac{R^{3} L\left(1-\sigma^{2}\right)}{\rho h(\varepsilon) E(\varepsilon)} \mathcal{P}^{2} \\
\int_{0}^{t}\left\{\left\|\frac{\partial v_{r}^{\varepsilon}}{\partial r}\right\|_{L^{2}\left(\Omega_{\varepsilon}(t)\right)}^{2}+\left\|\frac{v_{r}^{\varepsilon}}{r}\right\|_{L^{2}\left(\Omega_{\varepsilon}(t)\right)}^{2}+\left\|\frac{\partial v_{z}^{\varepsilon}}{\partial z}\right\|_{L^{2}\left(\Omega_{\varepsilon}(t)\right)}^{2}\right\} d \tau \leq \frac{R^{2}}{2 \mu} \sqrt{\frac{R\left(1-\sigma^{2}\right)}{h(\varepsilon) E(\varepsilon) \rho}} \mathcal{P}^{2} \\
\int_{0}^{t}\left\{\left\|\frac{\partial v_{z}^{\varepsilon}}{\partial r}\right\|_{L^{2}\left(\Omega_{\varepsilon}\right)}^{2}+\left\|\frac{\partial v_{r}^{\varepsilon}}{\partial z}\right\|_{L^{2}\left(\Omega_{\varepsilon}\right)}^{2}\right\} d \tau \leq \frac{R^{2}}{2 \mu} \sqrt{\frac{R\left(1-\sigma^{2}\right)}{h(\varepsilon) E(\varepsilon) \rho}} \mathcal{P}^{2}
\end{gathered}
$$


Proof. First notice that (4.10) and (4.11) are obvious consequences of (4.4). Next, (4.12) follows from

$$
\begin{gathered}
2 \pi \int_{0}^{t} \int_{0}^{L} \int_{0}^{R+\eta^{\varepsilon}}\left\{\left|\frac{\partial v_{r}^{\varepsilon}}{\partial r}\right|^{2}+\frac{1}{2}\left|\frac{\partial v_{z}^{\varepsilon}}{\partial r}+\frac{\partial v_{r}^{\varepsilon}}{\partial z}\right|^{2}+\left|\frac{\partial v_{z}^{\varepsilon}}{\partial z}\right|^{2}+\left(\frac{v_{r}^{\varepsilon}}{r}\right)^{2}\right\} r d r d z d \tau= \\
\int_{0}^{t}\left\|D\left(v^{\varepsilon}(t)\right)\right\|_{L^{2}\left(\Omega_{\varepsilon}(t)\right)}^{2} d \tau \leq \frac{\pi R^{2}}{\mu} \sqrt{\frac{R\left(1-\sigma^{2}\right)}{h(\varepsilon) E(\varepsilon) \rho}} \mathcal{P}^{2} .
\end{gathered}
$$

It remains to prove (4.13). We start from estimate (4.14) for the shear stress term in $D\left(v^{\varepsilon}\right)$. It reads

$$
\int_{0}^{t} \int_{0}^{L} \int_{0}^{R+\eta^{\varepsilon}}\left\{\left(\frac{\partial v_{r}^{\varepsilon}}{\partial z}\right)^{2}+2 \frac{\partial v_{r}^{\varepsilon}}{\partial z} \frac{\partial v_{z}^{\varepsilon}}{\partial r}+\left(\frac{\partial v_{z}^{\varepsilon}}{\partial r}\right)^{2}\right\} r d r d z d \tau \leq \frac{R^{2}}{\mu} \sqrt{\frac{R\left(1-\sigma^{2}\right)}{h(\varepsilon) E(\varepsilon) \rho}} \mathcal{P}^{2}
$$

The difficulty comes from the term which is the product of two off-diagonal gradient terms $\frac{\partial v_{r}^{\varepsilon}}{\partial z} \frac{\partial v_{z}^{\varepsilon}}{\partial r}$. We can estimate this term by using the boundary behavior of $v^{\varepsilon}$, $\partial_{z} v_{z}^{\varepsilon}=0$ at $z=0, L$, and the incompressibility condition (2.4) to obtain

$$
\begin{gathered}
\int_{\Omega_{\varepsilon}(t)} \frac{\partial v_{r}^{\varepsilon}}{\partial z} \frac{\partial v_{z}^{\varepsilon}}{\partial r} r d r d z=-\int_{\Omega_{\varepsilon}(t)} v_{z}^{\varepsilon} \frac{\partial}{\partial r}\left(r \frac{\partial v_{r}^{\varepsilon}}{\partial z}\right) d r d z \\
=\int_{\Omega_{\varepsilon}(t)} v_{z}^{\varepsilon} \frac{\partial^{2} v_{z}^{\varepsilon}}{\partial z^{2}} r d r d z=-\int_{\Omega_{\varepsilon}(t)} \frac{\partial}{\partial z} v_{z}^{\varepsilon} \frac{\partial v_{z}^{\varepsilon}}{\partial z} r d r d z=-\int_{\Omega_{\varepsilon}(t)}\left(\frac{\partial v_{z}^{\varepsilon}}{\partial z}\right)^{2} r d r d z .
\end{gathered}
$$

The rest of the proof is now immediate. $\square$

Corollary 4.4. We have

$$
\begin{gathered}
\frac{1}{L}\left\|\frac{\partial \eta^{\varepsilon}}{\partial z}(t)\right\|_{L^{2}(0, L)}^{2} \leq \frac{2 R^{2}\left(1-\sigma^{2}\right)}{G k h(\varepsilon)^{2} E(\varepsilon)} \mathcal{P}^{2} \\
\frac{1}{L^{1 / 4}\left\|\eta^{\varepsilon}(t)\right\|_{L^{4}(0, L)}} \leq \frac{2 R}{h(\varepsilon) E(\varepsilon)} \sqrt{R L\left(1-\sigma^{2}\right) \sqrt{\frac{E(\varepsilon)\left(1-\sigma^{2}\right)}{G k}}} \mathcal{P} \\
\left|\Omega_{\varepsilon}(t)\right| \leq \frac{3 \pi R^{2} L}{2}\left(1+\frac{2\left(1-\sigma^{2}\right) R^{2}}{h^{2}(\varepsilon) E(\varepsilon)^{2}} \mathcal{P}^{2}\right) \\
\left\|\eta^{\varepsilon}(t)\right\|_{L^{\infty}(0, L)} \leq \frac{2 R\left(1-\sigma^{2}\right)}{h(\varepsilon) E(\varepsilon)} \sqrt{R L\left(1-\sigma^{2}\right) \sqrt{\frac{E(\varepsilon)\left(1-\sigma^{2}\right)}{G k}}} \mathcal{P}
\end{gathered}
$$

Proof. Estimate (4.15) follows from the basic a priori estimate. To show (4.16) we calculate

$$
\left|\eta^{\varepsilon}(t, z)\right|^{4}=4\left(\int_{0}^{z} \eta^{\varepsilon}(t, \xi) \frac{\partial \eta^{\varepsilon}}{\partial \xi} d \xi\right)^{2} \leq 4\left(\int_{0}^{z}\left|\eta^{\varepsilon}(t, \xi)\right|^{2} d \xi\right)\left(\int_{0}^{z}\left|\frac{\partial \eta^{\varepsilon}}{\partial \xi}\right|^{2} d \xi\right)
$$

which implies $\int_{0}^{L}\left|\eta^{\varepsilon}(t, z)\right|^{4} d z \leq 4 L\left\|\frac{\partial \eta^{\varepsilon}}{\partial z}\right\|_{L^{2}(0, L)}^{2}\left\|\eta^{\varepsilon}(t)\right\|_{L^{2}(0, L)}^{2}$. Estimate (4.17) is immediate. Finally, (4.18) follows from

$$
\max _{0 \leq z \leq L}\left|\eta^{\varepsilon}(t, z)\right| \leq \sqrt{2}\left\|\frac{\partial \eta^{\varepsilon}}{\partial z}\right\|_{L^{2}(0, L)}^{1 / 2}\left\|\eta^{\varepsilon}(t)\right\|_{L^{2}(0, L)}^{1 / 2}
$$


Notice that these estimates are "reasonable". They say, among other things, that the size of the radial wall displacement is inversely proportional to the elasticity of the wall (the stiffer the wall, the smaller the amplitude of the displacement), and directly proportional to the pressure head data and to the radius of the unstressed vessel. In addition to this "general", intuitive information, our a priori estimates are "optimal" in the sense that they provide "optimal" powers describing the dependence of $\eta^{\varepsilon}$ and $v^{\varepsilon}$ on the parameters in the problem.

REMARK 4.5. Obtaining the precise a priori estimate for the pressure $p^{\varepsilon}$ is quite technical and we do not present it here. Since the flow is incompressible, pressure is a Lagrange multiplier and we can always adjust it with respect to the velocity. In the case of small Reynolds numbers such an estimate was obtained in [6]. The important property of the pressure estimates is the smallness of the derivative with respect to the radial variable.

REMARK 4.6. Our goal is to obtain an effective reduced model. Clearly, the result will be local and it does not depend on the choice of the inlet/outlet boundary conditions. We work with a given pressure head at the inlet/outlet boundaries solely to get a simple derivation of the energy estimate. Imposing a pressure field instead leads to technical complications and the energy estimate could be obtained only for the pressure drop smaller than a critical value.

\section{Asymptotic Expansions and the Reduced Equations.}

5.1. Leading-order asymptotic equations in non-dimensional form. To obtain the reduced equations we write the problem in non-dimensional form. Introduce the non-dimensional independent variables $\tilde{r}$ and $\tilde{z}$

$$
r=R \tilde{r}, \quad z=L \tilde{z},
$$

and recall that the time scale for the problem is determined by

$$
t=\frac{1}{\omega^{\varepsilon}} \tilde{t}, \text { where } \omega^{\varepsilon}=\frac{1}{L} \sqrt{\frac{h E}{R \rho\left(1-\sigma^{2}\right)}} .
$$

Using a range of data presented in Table 2.1, we obtain that $\omega^{\varepsilon}$ is between 438 and 113 and the time scale is between 0.002 and 0.008 of the physical time. For the less stiff vessels, the time-scale is closer to the physical time scale (the frequency of oscillations is smaller). Based on the a priori estimates presented in Proposition 4.3 we introduce the following asymptotic expansions

$$
\begin{aligned}
& v^{\varepsilon}=V\left\{\tilde{v}^{0}+\varepsilon \tilde{v}^{1}+\cdots\right\}, \text { with } V=\sqrt{\frac{R\left(1-\sigma^{2}\right)}{\rho h E}} \mathcal{P} \\
& \eta^{\varepsilon}=\Xi\left\{\tilde{\eta}^{0}+\varepsilon \tilde{\eta}^{1}+\cdots\right\}, \text { with } \Xi=\frac{R^{2}\left(1-\sigma^{2}\right)}{h E} \mathcal{P} \\
& p^{\varepsilon}=\rho V^{2}\left\{\tilde{p}^{0}+\varepsilon \tilde{p}^{1}+\cdots\right\} .
\end{aligned}
$$

The approximate values of the scaling parameters, based on the values presented in Table 2.1 with $E=6 \times 10^{5} \mathrm{~Pa}$ are $V=1 \mathrm{~m} / \mathrm{s}$ and $\Xi=0.0001 \mathrm{~m}$ for the iliac arteries and $V=2 \mathrm{~m} / \mathrm{s}$ and $\Xi=0.001 \mathrm{~m}$ for the abdominal aorta. The velocity values are in good agreement with the results in $[18,36]$. The scale value $\Xi$ of the radial displacement for the iliac arteries is about 4 percent and for the abdominal 
aorta about 13 percent, which is in good agreement with the radial displacement in human arteries which typically does not exceed 10 percent of the unstressed radius. We plug this into equations (2.2), (2.3) and (2.4) and collect the powers of $\varepsilon$. The incompressibility condition implies

$$
\varepsilon^{-1} \frac{\partial}{\tilde{r} \partial \tilde{r}}\left(\tilde{r} \tilde{v}_{\tilde{r}}^{0}\right)+\frac{\partial \tilde{v}_{z}^{0}}{\partial \tilde{z}}+\frac{\partial}{\tilde{r} \partial \tilde{r}}\left(\tilde{r} \tilde{v}_{\tilde{r}}^{1}\right)+\varepsilon \sum_{i \geq 0} \varepsilon^{i}\left\{\frac{\partial \tilde{v}_{\tilde{z}}^{i+1}}{\partial \tilde{z}}+\frac{\partial}{\tilde{r} \partial \tilde{r}}\left(\tilde{r} \tilde{v}_{\tilde{r}}^{i+2}\right)\right\}=0 .
$$

Relation (5.6) gives

$$
\begin{aligned}
& \tilde{v}_{\tilde{r}}^{0}=0, \quad \text { and } \\
& \frac{\partial\left(\tilde{v}_{\tilde{z}}^{0}+\varepsilon \tilde{v}_{\tilde{z}}^{1}\right)}{\partial \tilde{z}}+\frac{\partial}{\tilde{r} \partial \tilde{r}}\left(\tilde{r}\left(\tilde{v}_{\tilde{r}}^{1}+\varepsilon \tilde{v}_{\tilde{r}}^{2}\right)\right)=0 .
\end{aligned}
$$

Because the first term in the expansion for the radial component of the velocity is zero, and since only the first two terms in the dependent variable expansions will contribute to the leading order equations, we introduce the following notation

$$
\begin{gathered}
\tilde{v}_{r}:=\tilde{v}_{r}^{1}+\varepsilon \tilde{v}_{r}^{2}, \text { so that } v_{r}^{\varepsilon}=\varepsilon V\left(\tilde{v}_{r}+\mathcal{O}\left(\varepsilon^{2}\right)\right), \\
\tilde{v}_{z}:=\tilde{v}_{z}^{0}+\varepsilon \tilde{v}_{z}^{1} \text { so that } v_{z}^{\varepsilon}=V\left(\tilde{v}_{z}+\mathcal{O}\left(\varepsilon^{2}\right)\right), \\
\tilde{p}:=\tilde{p}^{0}+\varepsilon \tilde{p}^{1} \text { so that } p^{\varepsilon}=\rho V^{2}\left(\tilde{p}+\mathcal{O}\left(\varepsilon^{2}\right)\right), \\
\tilde{\eta}:=\tilde{\eta}^{0}+\varepsilon \tilde{\eta}^{1} \text { so that } \eta^{\varepsilon}=\Xi\left(\tilde{\eta}+\mathcal{O}\left(\varepsilon^{2}\right)\right) .
\end{gathered}
$$

After ignoring the terms of order $\varepsilon^{2}$ and smaller, the leading-order asymptotic equations describing the conservation of axial and radial momentum, and the incompressibility condition in non-dimensional variables read

$$
\begin{aligned}
S h \frac{\partial \tilde{v}_{z}}{\partial \tilde{t}}+\tilde{v}_{z} \frac{\partial \tilde{v}_{z}}{\partial \tilde{z}}+\tilde{v}_{r} \frac{\partial \tilde{v}_{z}}{\partial \tilde{r}}+\frac{\partial \tilde{p}}{\partial \tilde{z}}-\frac{1}{R e}\left\{\frac{1}{\left.\tilde{r} \frac{\partial}{\partial \tilde{r}}\left(\tilde{r} \frac{\partial \tilde{v}_{z}}{\partial \tilde{r}}\right)\right\}}=0,\right. \\
\frac{\partial \tilde{p}}{\partial \tilde{r}}=0, \\
\frac{\partial}{\partial \tilde{r}}\left(\tilde{r} \tilde{v}_{r}\right)+\frac{\partial}{\partial \tilde{z}}\left(\tilde{r} \tilde{v}_{z}\right)=0
\end{aligned}
$$

where $S h:=\frac{L \omega^{\varepsilon}}{V}$ and $R e:=\frac{\rho V R^{2}}{\mu L}$. Using the values from Table 2.1 our estimates imply that $S h$ ranges from $S h=23$ for iliac arteries to $S h=7$ for the abdominal aorta, and the values of $R e$ range between $R e=34$ in iliac arteries to $R e=295$ in the abdominal aorta. One interesting consequence of our results is that the non-dimensional parameters $S h$ and $R e$ which are typically used to determine the flow regimes, are given, as a consequence of our a priori estimates, in terms of the parameters in the problem, such as the Young's modulus, the Poisson ratio, etc. They incorporate not only the information about the fluid part of the problem (given via $V$ and $\mu$, for example) but also the information about the behavior of the membrane (given via $E$, $\omega^{\varepsilon}$ and $\sigma$ ). These parameters reflect the important information about the true nature of the coupling between the fluid and the membrane. We continue by obtaining the leading order asymptotic equations describing the balance of forces at the vessel wall. The leading order Navier equations for the linearly elastic membrane read

$$
-F_{r}=\mathcal{P}\left\{\tilde{\eta}-\frac{G(\varepsilon) k\left(1-\sigma^{2}\right) \varepsilon^{2}}{E(\varepsilon)} \frac{\partial^{2} \tilde{\eta}}{\partial \tilde{z}^{2}}\right\}+\mathcal{O}\left(\varepsilon^{2}\right)
$$


Since $G=E /(2(1+\sigma))$ in linear elasticity [11, 23] and $k=O(1)$, the second term on the right hand-size can also be neglected. This force is balanced by the contact force coming from the fluid. Using (2.9) the asymptotic form of the contact force becomes

$$
\left(\left(p^{\varepsilon}-p_{\text {ref }}\right) I-2 \mu D\left(v^{\varepsilon}\right)\right) \vec{n} \vec{e}_{r}=\rho V^{2}\left(\tilde{p}-\tilde{p}_{\text {ref }}+\mathcal{O}\left(\varepsilon^{2}\right)\right)\left(1+\frac{\Xi}{R} \tilde{\eta}\right) .
$$

Therefore, we obtain the following leading order relationship between the pressure and the radial displacement

$\tilde{p}^{\varepsilon}-\tilde{p}_{\text {ref }}=\frac{\mathcal{P}}{\rho V^{2}} \frac{\tilde{\eta}}{1+\frac{\Xi}{R} \tilde{\eta}}=\frac{\mathcal{P} R}{\rho V^{2} \Xi} \frac{\Xi}{R} \tilde{\eta}\left(1-\frac{\Xi}{R} \tilde{\eta}+\cdots\right)=\frac{\mathcal{P} R}{\rho V^{2} \Xi}\left(\frac{\Xi}{R} \tilde{\eta}-\left(\frac{\Xi}{R} \tilde{\eta}\right)^{2}+\cdots\right)$

If we assume that $\Xi / R$ is of order $\epsilon$, which is consistent with the assumptions of linear elasticity used to describe the vessel wall behavior, then the last term on the righthand side can be ignored and we get a linear contact force relationship. In nonlinear models, such as those studied in [7], nonlinear contact force relationship is appropriate and the term $(1+\Xi / R \tilde{\eta})$ is kept. In the present manuscript we continue in the spirit of linear elasticity and consider the cases when $\Xi / R \leq \epsilon$. Table 2.1 implies that for the data corresponding to iliac arteries $\Xi / R$ is indeed of order $\epsilon$. A discussion on the (true) size of the scaling constant between the pressure and the radial displacement is presented in $\S 7$ after equation (7.7) where the improved scaling for the effective pressure corresponding to our flow regime is used.

Assuming linear contact force relationship we obtain

$$
\tilde{p}-\tilde{p}_{\text {ref }}=\frac{\mathcal{P}}{\rho V^{2}} \tilde{\eta}
$$

which in dimensional variables gives the Law of Laplace

$$
p-p_{\text {ref }}=\left(\frac{E h}{\left(1-\sigma^{2}\right) R}\right) \frac{\eta}{R} .
$$

5.2. The reduced two-dimensional coupled problem. We summarize here the two-dimensional reduced coupled problem in non-dimensional variables. Define the scaled domain

$$
\tilde{\Omega}(\tilde{t})=\left\{(\tilde{z}, \tilde{r}) \in \mathbb{R}^{2} \mid \tilde{r}<1+\frac{\Xi}{R} \tilde{\eta}(\tilde{z}, \tilde{t}), 0<\tilde{z}<1\right\},
$$

and the lateral boundary $\tilde{\Sigma}(\tilde{t})=\left\{\tilde{r}=1+\frac{\Xi}{R} \tilde{\eta}(\tilde{z}, \tilde{t})\right\} \times(0,1)$. The problem consist of finding a $\left(\tilde{v}_{z}, \tilde{v}_{r}, \tilde{\eta}\right)$ such that in $\tilde{\Omega}(\tilde{t}) \times \mathbb{R}^{+}$the following is satisfied

$$
\begin{gathered}
S h \frac{\partial \tilde{v}_{z}}{\partial \tilde{t}}+\tilde{v}_{z} \frac{\partial \tilde{v}_{z}}{\partial \tilde{z}}+\tilde{v}_{r} \frac{\partial \tilde{v}_{z}}{\partial \tilde{r}}+\frac{\partial \tilde{p}}{\partial \tilde{z}}=\frac{1}{R e}\left\{\frac{1}{\tilde{r}} \frac{\partial}{\partial \tilde{r}}\left(\tilde{r} \frac{\partial \tilde{v}_{z}}{\partial \tilde{r}}\right)\right\} \\
\frac{\partial}{\partial \tilde{r}}\left(\tilde{r} \tilde{v}_{r}\right)+\frac{\partial}{\partial \tilde{z}}\left(\tilde{r} \tilde{r}_{z}\right)=0 \\
\tilde{p}-\tilde{p}_{\mathrm{ref}}=\frac{\mathcal{P}}{\rho V^{2}} \tilde{\eta}, \\
\tilde{v}_{r}\left(\tilde{z}, 1+\frac{\Xi}{R} \tilde{\eta}(z, t), \tilde{t}\right)=\frac{\partial \tilde{\eta}}{\partial \tilde{t}}, \quad \tilde{v}_{z}=0
\end{gathered}
$$


with the initial and boundary conditions given by

$$
\begin{aligned}
& \tilde{\eta}=\frac{\partial \tilde{\eta}}{\partial \tilde{t}}=0 \quad \text { at }\{\tilde{t}=0\}, \\
& \tilde{v}_{\tilde{r}}=0 \text { and } \tilde{p}=\left(P_{1}(\tilde{t})+p_{\mathrm{ref}}\right) /\left(\rho V^{2}\right) \text { on } \quad(\partial \tilde{\Omega}(\tilde{t}) \cap\{\tilde{z}=0\}) \times \mathbb{R}_{+}, \\
& \tilde{v}_{\tilde{r}}=0 \text { and } \tilde{p}=\left(P_{2}(\tilde{t})+p_{\mathrm{ref}}\right) /\left(\rho V^{2}\right) \quad \text { on } \quad(\partial \tilde{\Omega}(\tilde{t}) \cap\{\tilde{z}=1\}) \times \mathbb{R}_{+}, \\
& \tilde{\eta}=0 \text { for } \tilde{z}=0, \quad \tilde{\eta}=0 \text { for } \tilde{z}=1 \text { and } \forall \tilde{t} \in \mathbb{R}_{+} .
\end{aligned}
$$

This is a closed, free-boundary problem for a two-dimensional degenerate hyperbolic system with a parabolic regularization. Its solution is difficult to study both theoretically and numerically. This is why we continue by simplifying this problem even further. First we present a one-dimensional approximation of problem (5.15)-(5.22) obtained using averaging, which requires an ad hoc closure assumption on the axial velocity profile. We show that we recover the standard one-dimensional hyperbolic system used in $[3,4,10,16,26,28,34,38]$. Then, in $\S 7$ we obtain a simplified effective system without an ad hoc closure for which we prove that it solves problem (5.15)-(5.22) to the $\epsilon^{2}$ accuracy. In Appendix B we show the existence, uniqueness and regularity of the resulting effective equations and in $\S 8$ we show numerical simulations of the new effective equations.

REMARK 5.1. We do not discuss here the asymptotic behavior close to the inlet/outlet boundaries. With our data the term $\rho\left(v_{z}^{0}\right)^{2} / 2$ is negligible compared to the pressure and we ignore it.

6. The Averaged Equations. To simplify the problem even further and obtain the effective equations in one space dimension we use a typical approach of averaging the two-dimensional equations across the vessel cross-section. Introduce $\tilde{A}=\left(1+\frac{\Xi}{R} \tilde{\eta}\right)^{2}$ and $\tilde{m}=\tilde{A} \tilde{U}$ where

$$
\tilde{U}=\frac{2}{\tilde{A}} \int_{0}^{1+\frac{\overline{\bar{V}}}{R} \tilde{\eta}} \tilde{v}_{z} \tilde{r} d \tilde{r} \quad \text { and } \quad \tilde{\alpha}=\frac{2}{\tilde{A} \tilde{U}^{2}} \int_{0}^{1+\frac{\bar{E}}{R} \tilde{\eta}} \tilde{v}_{z}^{2} \tilde{r} d \tilde{r}
$$

We integrate the incompressibility condition and the axial momentum equations with respect to $\tilde{r}$ from 0 to $1+\frac{\Xi}{R} \tilde{\eta}$ and obtain, after taking into account the no-slip condition at the lateral boundary,

$$
\begin{gathered}
\frac{\partial \tilde{A}}{\partial \tilde{t}}+\frac{\Xi}{R} \frac{\partial \tilde{m}}{\partial \tilde{z}}=0 \\
S h \frac{\partial \tilde{m}}{\partial \tilde{t}}+\frac{\partial}{\partial \tilde{z}}\left(\tilde{\alpha} \frac{\tilde{m}^{2}}{\tilde{A}}\right)+\tilde{A} \frac{\partial \tilde{p}}{\partial \tilde{z}}=\frac{2}{R e} \sqrt{\tilde{A}}\left[\frac{\partial \tilde{v}_{z}}{\partial \tilde{r}}\right]_{\tilde{\Sigma}} .
\end{gathered}
$$

As always when averaging nonlinear systems one needs a closure condition. In our case this amounts to describing the axial velocity profile. There are several ad hoc approaches in literature. They assume the Poiseuille velocity profile

$$
\tilde{v}_{z}=\frac{\gamma+2}{\gamma} \tilde{U}\left(1-\left(\frac{\tilde{r}}{1+\frac{\Xi}{R} \tilde{\eta}}\right)^{\gamma}\right)
$$

where $\gamma=2$, an "almost flat" velocity profile corresponding to (6.3) with $\gamma=9$ which accounts for the non-Newtonian nature of blood [34], the flat velocity profile ("plug flow"), or the flat velocity profile with a small linear boundary layer (Bingham 
flow) suggested in [28]. In order to compare our results thus far with those already existing in the literature, in this section we assume one of the ad hoc velocity profiles mentioned above, namely the profile given by (6.3) with $\gamma=9$. This gives rise to $\alpha=1.1$. With this assumption, the term on the right hand-side of the momentum equation becomes

$$
-\frac{2}{R e}(\gamma+2) \frac{\tilde{m}}{\tilde{A}}
$$

The pressure term is specified by (5.13). Using $A_{0}$ to denote the non-stressed area $R^{2}$ we obtain the following system written in dimensional variables

$$
\begin{gathered}
\frac{\partial A}{\partial t}+\frac{\partial m}{\partial z}=0 \\
\frac{\partial m}{\partial t}+\frac{\partial}{\partial z}\left(\alpha \frac{m^{2}}{A}\right)+\frac{A}{\rho} \frac{\partial}{\partial z}\left(\frac{h E}{R\left(1-\sigma^{2}\right)}\left(\sqrt{\frac{A}{A_{0}}}-1\right)\right)=-\frac{2 \mu}{\rho}(\gamma+2) \frac{m}{A} .
\end{gathered}
$$

For $\sigma=0.5$ this is the quasilinear hyperbolic system widely used in literature [3, 4, 10, 16, 26, 28, 34, 38].

7. An $\varepsilon^{2}$-approximation without an ad hoc closure assumption. In this section we obtain the one-dimensional, closed, effective equations that are an $\varepsilon^{2}$ approximation of the original 3-D axially symmetric fluid-structure interaction problem. The equations are simpler than those presented in (5.9)-(5.12). They can easily be solved numerically. We consider two flow regimes. One is the creeping flow regime typical for coronary arteries [28], discussed in $\$ 7.1$, and the other is the flow regime typical for iliac arteries and the abdominal aorta, namely moderate Reynolds number, discussed in $\$ 7.2$. In the creeping flow regime, it is well known that the Poiseuille profile is the unique velocity solution to the stationary equations. We show that the displacement is described by a one-dimensional, parabolic, semi-linear equation, see (7.11), first obtained by Čanić and Mikelić in $[5,6]$. In the moderate Reynolds' number regime, we assume that $\Xi / R \leq \epsilon$ which is consistent with the assumptions of linear elasticity that we use to describe the behavior of vessel walls. We expand the solution in terms of the small parameter $\Xi / R$ and obtain a linear system of equations of Biot type, see [1]. This is obtained in $\S 7.2 .5$. These equations can be easily solved using, for example, the Laplace transform, see Appendix A. We obtain that for a time-periodic flow regime the resulting velocity profiles are an $\varepsilon$-order correction of the Womersley profile in elastic tubes [39]. We begin our analysis by studying the two-dimensional system (5.9)-(5.11). Our goal is to obtain an equivalent effective problem which is closed, and for which we could show the existence of a unique solution. Furthermore, the calculation of the solution for such a system should be simple. Motivated by the results of [22] where closed effective porous medium equations were obtained using homogenization techniques, we would like to set up a problem that would mimic a similar scenario. A typical approach in homogenization of porous media flows is to take into account periodic structure of the underlying domain, such as the one shown in Figure 7.1 on the right, and look for a solution which exhibits oscillations determined by the scale, say $2 \epsilon$, at which the domain repeats itself periodically. To capture the oscillations occurring at the small scale (fast oscillations), one looks for a solution, say $v$, which depends on both the global slow variables as well as on the "fast" variables. If $r$ and $\tilde{z}$ are the global spatial variables then one looks for a function $v(t, r, \tilde{z})=\tilde{v}(\tilde{t}, r, r / \epsilon, \tilde{z}, \tilde{z} / \epsilon)$ that explicitly depends on the slow, 


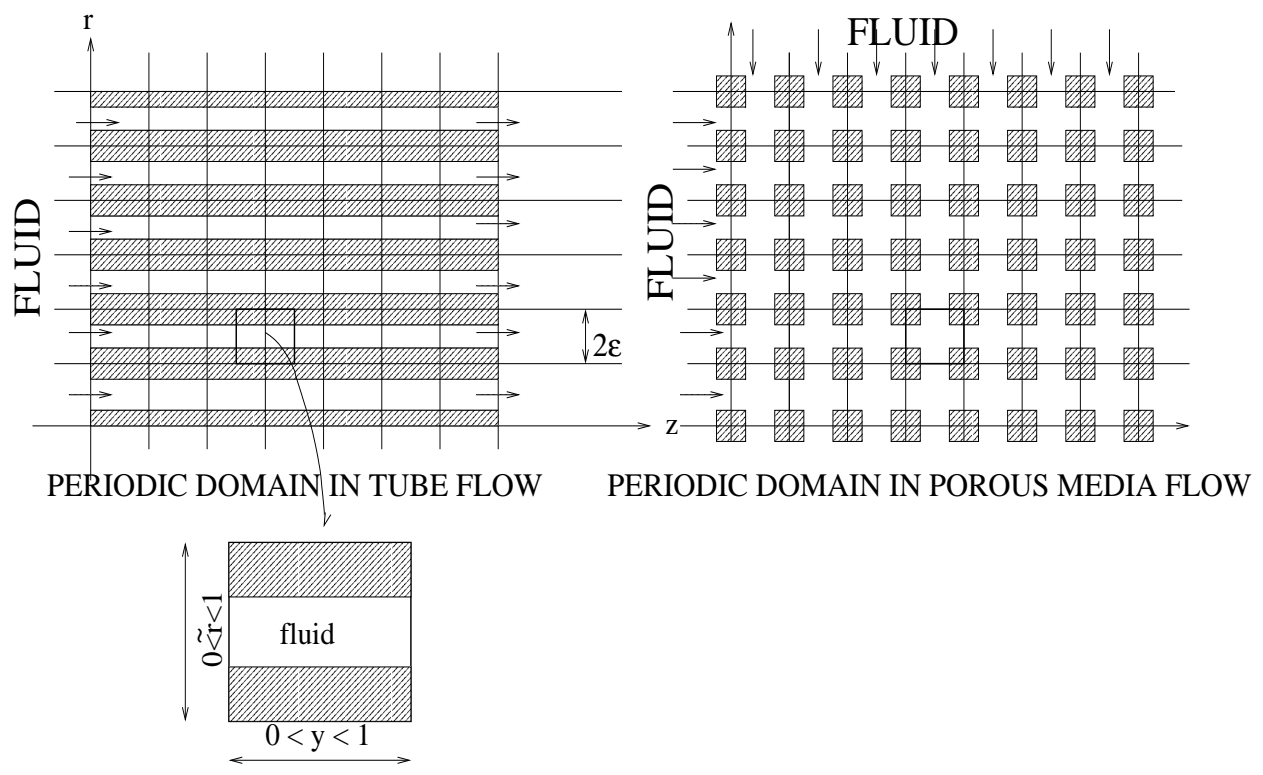

FIG. 7.1. Homogenization domains.

as well as on the "fast" variables $r / \epsilon$ and $\tilde{z} / \epsilon$. In our case we have a "natural" small parameter $\epsilon$ that defines a fast scale $r / \epsilon=\tilde{r}$. By periodically extending our domain in the radial direction, see Figure 7.1 on the left, we can think of our problem as a porous medium problem which consists of studying a flow through a network of a large number of strictly separated, parallel tubes, shown in Figure 7.1 on the left. Now, even though there is no flow in the transverse direction we can still introduce a "fictive" fast variable $y=\tilde{z} / \varepsilon$ and assume periodicity in $y$ of the domain and of the velocity and pressure. This should not affect the solution, but, as we will see below, it will help us obtain a closed set of equations. The primary reasons for that are two-fold: one is the fact that the higher-order terms resulting from equations (5.9)-(5.11) that need additional conditions for closure are all given in terms of the pressure, and the second is that an $\epsilon^{2}$ approximation of equations (5.9)-(5.11) is a hydrostatic one (the pressure is independent of the radial variable). Therefore, the higher order terms will depend only on the "transverse fast variable" $y$ (and $\tilde{z}$ ) in which the periodicity and "fictive dependence" on that variable will allow us to show that the higher-order terms are, in fact, all equal to zero. Once this is established, we homogenize with respect to all directions and obtain a closed system of effective equations, presented in $\$ 7.2 .3$ and $\S 7.2 .5$. We note that thanks to the fact that the model contains a hydrostatic approximation of the pressure in straight tubes, the methods we use in this paper are much simpler than those used in [22]. For more details about the homogenization methods in porous media, see e.g. [24]. We start with the following relations between the "slow" variables $(r$ and $\tilde{z})$ and "fast" variables $(\tilde{r}$ and $y)$

$$
z=L \tilde{z}:=L \varepsilon y=R y, \quad r=R \tilde{r} .
$$

We are looking for an $\varepsilon^{2}$-approximation of the solution to system (5.9)-(5.11) in the form of a sum of two functions: the zero and the first order approximations with respect to $\varepsilon$. We use scaling (7.1) and dependent variable expansions given in (5.3) and (5.4) and plug them into equations (2.2)-(2.4). The equations at the zero-th order 
read

$$
\begin{gathered}
S h_{0} \frac{\partial \tilde{v}_{z}^{0}}{\partial \tilde{t}}+\left(\tilde{v}^{0} \nabla_{\tilde{r}, y}\right) \tilde{v}_{z}^{0}+\frac{\partial \tilde{\tilde{p}}^{0}}{\partial \tilde{z}}+\frac{\partial \tilde{\tilde{p}}^{1}}{\partial y}-\frac{1}{R e_{0}}\left\{\frac{1}{\tilde{r}} \frac{\partial}{\partial \tilde{r}}\left(\tilde{r} \frac{\partial \tilde{v}_{z}^{0}}{\partial \tilde{r}}\right)+\frac{\partial^{2} \tilde{v}_{z}^{0}}{\partial y^{2}}\right\}=0, \\
S h_{0} \frac{\partial \tilde{v}_{r}^{0}}{\partial \tilde{t}}+\left(\tilde{v}^{0} \nabla_{\tilde{r}, y}\right) \tilde{v}_{r}^{0}+\frac{\partial \tilde{\tilde{p}}^{0}}{\partial r}+\frac{\partial \tilde{\tilde{p}}^{1}}{\partial \tilde{r}}-\frac{1}{R e_{0}}\left\{\frac{1}{\tilde{r}} \frac{\partial}{\partial \tilde{r}}\left(\tilde{r} \frac{\partial \tilde{v}_{r}^{0}}{\partial \tilde{r}}\right)+\frac{\partial^{2} \tilde{v}_{r}^{0}}{\partial y^{2}}\right\}=0, \\
\nabla_{\tilde{r}, y} \tilde{\tilde{p}}^{0}=0, \\
\frac{\partial}{\partial \tilde{r}}\left(\tilde{r} \tilde{v}_{r}^{0}\right)+\frac{\partial}{\partial y}\left(\tilde{r} \tilde{v}_{z}^{0}\right)=0,
\end{gathered}
$$

with

$$
\tilde{v}_{r}^{0}, \tilde{v}_{z}^{0} \text { and } \tilde{\tilde{p}}^{1} 1-\text { periodic in } y \text { and } \tilde{v}_{r}^{0}=\tilde{v}_{z}^{0}=0 \text { at } \tilde{r}=1+\frac{\Xi}{R} \tilde{\eta},
$$

where $S h_{0}:=\frac{\varepsilon L \omega^{\varepsilon}}{V}$ and $R e_{0}:=\frac{\rho R V}{\mu}$. Notice $S h_{0}=\varepsilon S h$ and $R e_{0}=R e / \varepsilon$. For the values from Table 2.1 corresponding to iliac arteries, $S h_{0}$ is of order 1 and $R e_{0}$ is around 900. We remark that equation (7.4) corresponds to the $\varepsilon^{-1}$ term. Here, a new scaling for the pressure was used to obtain equations (7.2)-(7.4):

$$
p=\frac{\rho L V^{2}}{R} \tilde{\tilde{p}}=\rho V^{2} \frac{1}{\varepsilon} \tilde{\tilde{p}}=\rho V^{2} \tilde{p}, \quad \text { so } \quad \tilde{\tilde{p}}=\varepsilon \tilde{p} .
$$

The leading order Navier equations for the membrane force are unchanged, see (5.12). We then have

$$
\tilde{\tilde{p}}-\tilde{\tilde{p}}_{\text {ref }}=\frac{\mathcal{P} R}{\rho V^{2} L} \frac{\tilde{\eta}}{1+\frac{\Xi}{R} \tilde{\eta}}=\frac{\mathcal{P} R^{2}}{\rho V^{2} L \Xi}\left(\frac{\Xi}{R} \tilde{\eta}-\left(\frac{\Xi}{R} \tilde{\eta}\right)^{2}+\cdots\right)
$$

and since $\frac{\mathcal{P} R^{2}}{\rho V^{2} L \Xi}=O(1)$ and $\Xi / R \leq \epsilon$, using linear contact force relationship is justified. We now focus on two cases corresponding to the different magnitudes of the parameters $S h_{0}$ and $R e_{0}$.

7.1. Case I: $S h_{0}=0$ and $R e_{0}$ sufficiently small. In this case the Poiseuille profile

$$
\tilde{v}_{z}^{0}=-\operatorname{Re}_{0} \frac{\partial \tilde{\tilde{p}}}{\partial \tilde{z}}(\tilde{z}, \tilde{t}) \frac{\left(1+\Xi \tilde{\eta}^{0} / R\right)^{2}-\tilde{r}^{2}}{4}, \tilde{v}_{r}^{0}=0
$$

is the unique velocity which solves (7.2)-(7.6). To complete the solution we need to calculate the pressure and the radial displacement. They are related through the coupling at the lateral boundary

$$
\tilde{\tilde{p}}-\tilde{\tilde{p}}_{\text {ref }}=\frac{\mathcal{P} R}{\rho V^{2} L} \tilde{\eta}=\frac{h E}{\left(1-\sigma^{2}\right) L \mathcal{P}} \tilde{\eta} .
$$

Again, we are assuming that the shear modulus term is negligible and that linear coupling between the contact forces is appropriate. We average the continuity equation

$$
\frac{\partial}{\partial \tilde{r}}\left(\tilde{r} \tilde{v}_{r}^{0}\right)+\varepsilon \frac{\partial}{\partial \tilde{r}}\left(\tilde{r} \tilde{v}_{r}^{1}\right)+\varepsilon \frac{\partial}{\partial \tilde{z}}\left(\tilde{r} \tilde{v}_{z}^{0}\right)+\varepsilon^{2} \frac{\partial}{\partial \tilde{z}}\left(\tilde{r} \tilde{v}_{r}^{1}\right)=0
$$


keeping in mind that $\tilde{v}_{r}^{0}=0, \tilde{v}_{r}^{1}=\partial \tilde{\eta}^{0} / \partial \tilde{t}$, and ignoring the term at $\varepsilon^{2}$. As before, we get the following

$$
\frac{\partial \tilde{A}}{\partial \tilde{t}}+\frac{\Xi}{R} \frac{\partial \tilde{m}}{\partial \tilde{z}}=0
$$

where $\tilde{A}=\left(1+\Xi \tilde{\eta}^{0} / R\right)^{2}$. Express $\tilde{m}$ explicitly using the Poiseuille velocity profile (7.8), namely

$$
\tilde{m}=\tilde{A} \tilde{U}=2 \int_{0}^{1+\Xi \tilde{\eta}^{0} / R} \tilde{r} \tilde{v}_{z}^{0} d \tilde{r}
$$

to obtain the following semilinear parabolic equation for the cross-sectional area

$$
\frac{\partial \tilde{A}}{\partial \tilde{t}}=\frac{R e_{0}}{8} \frac{R}{\rho V L} \sqrt{\frac{h E \rho}{R\left(1-\sigma^{2}\right)}} \frac{\partial}{\partial \tilde{z}}\left(\tilde{A}^{2} \frac{\partial \sqrt{\tilde{A}}}{\partial \tilde{z}}\right) .
$$

In dimensional variables this reads

$$
8 \mu \frac{\partial A}{\partial t}=\frac{h E}{R^{2}\left(1-\sigma^{2}\right)} \frac{\partial}{\partial z}\left(A^{2} \frac{\partial \sqrt{A}}{\partial z}\right) .
$$

This is a semi-linear variant of the equations obtained by Čanić and Mikelić in [6], where a parabolic equation for the pressure was obtained. The effective equation holds in axi-symmetric domains, and they are an $\varepsilon^{2}$-approximation of the 3-D axially symmetric flow away from the boundary. There was no ad hoc closure assumption made on the form of the velocity profile. We proceed in the same spirit, but for a more complicated scenario.

7.2. CASE II: $S h_{0}>0$ AND moderate $R e_{0}$. In this case, for a given pressure gradient $\frac{\partial \tilde{\tilde{p}} \tilde{p}^{0}}{\partial \tilde{z}}$, the non-stationary, axially symmetric system (7.2)-(7.6) admits a unique unidirectional, but strongly non-stationary solution. The unidirectional solution refers to a solution independent of $y$. We will write the solution of system (7.2)-(7.6) as a sum of this unidirectional solution and a small perturbation of it. This perturbation satisfies a linearized system, see (7.2)-(7.6), where the linearization is calculated around the unidirectional solution. This system is closed.

7.2.1. The zero-th order approximation: the unidirectional flow. For every given smooth $\tilde{\tilde{p}}^{0}$, system (7.2)-(7.6) has a unique strong solution (see e.g., [37])

$$
\tilde{v}_{z}^{0}=w(\tilde{r}, \tilde{z}, t), \tilde{v}_{r}^{0}=0,
$$

where $w$ satisfies

$$
\begin{gathered}
S h_{0} \frac{\partial w}{\partial \tilde{t}}-\frac{1}{R e_{0}} \frac{1}{\tilde{r}} \frac{\partial}{\partial \tilde{r}}\left(\tilde{r} \frac{\partial w}{\partial \tilde{r}}\right)=-\frac{\partial \tilde{\tilde{p}}}{\partial \tilde{z}}(\tilde{z}, \tilde{t})=-\left(\frac{E h}{\mathcal{P} L\left(1-\sigma^{2}\right)}\right) \frac{\partial}{\partial \tilde{z}}\left(\frac{\tilde{\eta}^{0}}{1+\frac{\Xi}{R} \tilde{\eta}^{0}}\right) \\
w(0, \tilde{z}, \tilde{t}) \text { bounded, } w\left(1+\Xi \tilde{\eta}^{0}(\tilde{z}, \tilde{t}) / R, \tilde{z}, \tilde{t}\right)=0 \text { and } w(\tilde{r}, \tilde{z}, 0)=0
\end{gathered}
$$

Furthermore, solution $\tilde{p}^{1}$ is a linear function of $y$, independent of $\tilde{r}$. Due to 1periodicity with respect to $y$ we get $\tilde{p}^{1}=\tilde{p}^{1}(\tilde{z}, \tilde{t})$. This is a free-boundary problem 
because the condition at the lateral boundary depends on the solution. For a known pressure or the radial displacement (or the cross-sectional area) this is a well-posed problem for $w$. However, to close the system for the unknown functions $w, \tilde{\tilde{p}}^{0}$ and $\tilde{\eta}^{0}$ we need to specify one more condition. The averaged continuity equation provides the necessary closure. Therefore, the following closed system provides the unidirectional solution

$$
\begin{aligned}
\frac{\partial \tilde{A}}{\partial \tilde{t}}+\frac{\Xi}{R} \frac{\partial \tilde{m}}{\partial \tilde{z}} & =0, \\
S h_{0} \frac{\partial w}{\partial \tilde{t}}+\frac{\partial \tilde{\tilde{p}}}{\partial \tilde{z}}(\tilde{z}, \tilde{t}) & =\frac{1}{R e_{0}} \frac{1}{\tilde{r}} \frac{\partial}{\partial \tilde{r}}\left(\tilde{r} \frac{\partial w}{\partial \tilde{r}}\right), \quad \frac{\partial \tilde{\tilde{p}}^{0}}{\partial \tilde{z}}=\left(\frac{E h}{\mathcal{P} L\left(1-\sigma^{2}\right)}\right) \frac{\partial}{\partial \tilde{z}}\left(\frac{\tilde{\eta}^{0}}{1+\frac{\Xi}{R} \tilde{\eta}^{0}}\right)
\end{aligned}
$$

with $w(0, \tilde{z}, \tilde{t})$ bounded, $w\left(1+\Xi \tilde{\eta}^{0}(\tilde{z}, \tilde{t}) / R, \tilde{z}, \tilde{t}\right)=0$ and $w(\tilde{r}, \tilde{z}, 0)=0$. We can eliminate $\tilde{\tilde{p}}$, and use the definitions of $\tilde{A}$ and $\tilde{m}$ to write this in terms of $w$ and $\tilde{A}$ as

$$
\begin{gathered}
\frac{\partial \tilde{A}}{\partial \tilde{t}}+\frac{\Xi}{R} \frac{\partial}{\partial \tilde{z}} \int_{0}^{\sqrt{\tilde{A}}} 2 \tilde{r} w d \tilde{r}=0 \\
S h_{0} \frac{\partial w}{\partial \tilde{t}}+\left(\frac{R}{\Xi}\right)^{2} \frac{R}{L} \frac{\partial}{\partial \tilde{z}}\left(1-\sqrt{\frac{1}{\tilde{A}}}\right)=\frac{1}{R e_{0}} \frac{1}{\tilde{r}} \frac{\partial}{\partial \tilde{r}}\left(\tilde{r} \frac{\partial w}{\partial \tilde{r}}\right),
\end{gathered}
$$

with

$$
\begin{aligned}
w(0, \tilde{z}, \tilde{t}) \text { bounded, } w(\sqrt{\tilde{A}}, \tilde{z}, \tilde{t}) & =0 \\
\tilde{A}(\tilde{z}, 0)=1, \quad w(\tilde{r}, \tilde{z}, 0) & =0 \\
\tilde{A}(0, \tilde{t})=A_{1}(\tilde{t}), \quad \tilde{A}(L, \tilde{t}) & =A_{2}(\tilde{t}) .
\end{aligned}
$$

7.2.2. The first-order correction: perturbation of the unidirectional flow. We will be using the zero-th order approximation to the solution consisting of the velocity $(w, 0)$ and displacement $\tilde{\eta}^{0}$ (or, equivalently, the pressure $\tilde{\tilde{p}}^{0}$ ) to find an $\varepsilon$ correction by solving (5.9)-(5.11), linearized around the zero-th order approximation:

$$
\begin{gathered}
S h_{0} \frac{\partial \tilde{v}_{z}^{1}}{\partial \tilde{t}}+\tilde{v}_{z}^{0}\left\{\frac{\partial \tilde{v}_{z}^{1}}{\partial y}+\frac{\partial \tilde{v}_{z}^{0}}{\partial \tilde{z}}\right\}+\tilde{v}_{r}^{1} \frac{\partial \tilde{v}_{z}^{0}}{\partial \tilde{r}}+\frac{\partial \tilde{\tilde{p}}^{1}}{\partial \tilde{z}}+\frac{\partial \tilde{\tilde{p}}^{2}}{\partial y}=\frac{1}{R e_{0}}\left\{\frac{1}{\tilde{r}} \frac{\partial}{\partial \tilde{r}}\left(\tilde{r} \frac{\partial \tilde{v}_{z}^{1}}{\partial \tilde{r}}\right)+\frac{\partial^{2} \tilde{v}_{z}^{1}}{\partial y^{2}}\right\} \\
S h_{0} \frac{\partial \tilde{v}_{r}^{1}}{\partial \tilde{t}}+\tilde{v}_{z}^{0} \frac{\partial \tilde{v}_{r}^{1}}{\partial y}+\frac{\partial \tilde{\tilde{p}}^{2}}{\partial \tilde{r}}=\frac{1}{R e_{0}}\left\{\frac{1}{\tilde{r}} \frac{\partial}{\partial \tilde{r}}\left(\tilde{r} \frac{\partial \tilde{v}_{r}^{1}}{\partial \tilde{r}}\right)+\frac{\partial^{2} \tilde{v}_{r}^{1}}{\partial y^{2}}\right\} \\
\frac{\partial}{\partial \tilde{r}}\left(\tilde{r} \tilde{v}_{r}^{1}\right)+\frac{\partial}{\partial y}\left(\tilde{r} \tilde{v}_{z}^{1}\right)+\tilde{r} \frac{\partial \tilde{v}_{z}^{0}}{\partial \tilde{z}}=0, \\
\tilde{v}_{r}^{1}, \tilde{v}_{z}^{1}, \tilde{\tilde{p}}^{2} 1-\text { periodic in } y ; \tilde{v}_{r}^{1}=\frac{\partial \tilde{\eta}^{0}}{\partial \tilde{t}}, \tilde{v}_{z}^{0}=0 \text { at } \tilde{r}=1+\frac{\Xi}{R} \tilde{\eta}^{0} .
\end{gathered}
$$

This is a linear system which is known as a non-stationary Oseen's system. Since $\tilde{\eta}^{0}$ is known from the previous step, the problem is posed on a fixed cylindrical domain of radius $\tilde{r}=1+\frac{\Xi}{R} \tilde{\eta}^{0}$. Notice, however, that the system does not appear to be closed since $\tilde{\tilde{p}}^{1}=\tilde{\tilde{p}}^{1}(\tilde{z}, \tilde{t})$ and $\tilde{\tilde{p}}^{2}=\tilde{\tilde{p}}^{2}(\tilde{r}, y, \tilde{z}, \tilde{t})$ are unknown as well. Nevertheless, since $\tilde{\tilde{p}}^{1}=\tilde{\tilde{p}}(\tilde{z}, \tilde{t})$ is zero at the boundary $\tilde{r}=1+\Xi \tilde{\eta}^{0} / R$ and it depends only on $(\tilde{z}, \tilde{t}), \tilde{p}^{1}$ 
must be zero. We will show below that $\tilde{\tilde{p}}^{2}=0$ which will lead to a closed system. To show that this is, indeed, the case we first suppose that $\tilde{v}_{z}^{1}=\tilde{v}_{z}^{1}(\tilde{r}, \tilde{z}, \tilde{t})$ and calculate $\tilde{v}_{r}^{1}$ using (7.23). We get an explicit formula for $\tilde{v}_{r}^{1}(\tilde{r}, \tilde{z}, \tilde{t})$ in terms of the unidirectional solution

$$
\tilde{r} \tilde{v}_{r}^{1}(\tilde{r}, \tilde{z}, \tilde{t})=\left(1+\Xi \tilde{\eta}^{0} / R\right) \frac{\partial \tilde{\eta}^{0}}{\partial \tilde{t}}+\int_{\tilde{r}}^{1+\Xi \tilde{\eta}^{0} / R} \frac{\partial \tilde{v}_{z}^{0}}{\partial \tilde{z}}(\xi, \tilde{z}, \tilde{t}) \xi d \xi
$$

Next using (7.22) we find $\tilde{\tilde{p}}^{2}$ in the form $\tilde{\tilde{p}}^{2}=\alpha(\tilde{r}, \tilde{z}, \tilde{t})+\varphi(y, \tilde{z}, \tilde{t})$, where $\varphi$ is an arbitrary function, 1-periodic in $y$. If we plug this into the axial momentum equation for $\tilde{v}_{z}^{1}$

$$
\begin{gathered}
S h_{0} \frac{\partial \tilde{v}_{z}^{1}}{\partial \tilde{t}}-\frac{1}{R e_{0}} \frac{1}{\tilde{r}} \frac{\partial}{\partial \tilde{r}}\left(\tilde{r} \frac{\partial \tilde{v}_{z}^{1}}{\partial \tilde{r}}\right)+\frac{\partial \varphi}{\partial y}(y, \tilde{z}, \tilde{t})=-\tilde{v}_{r}^{1} \frac{\partial \tilde{v}_{z}^{0}}{\partial \tilde{r}}-\frac{\partial}{\partial \tilde{z}}\left(\frac{\left(\tilde{v}_{z}^{0}\right)^{2}}{2}+\tilde{\tilde{p}}^{1}\right) \\
\tilde{v}_{z}^{1}(0, \tilde{z}, \tilde{t}) \text { bounded, } \tilde{v}_{z}^{1}\left(1+\Xi \tilde{\eta}^{0}(\tilde{z}, \tilde{t}) / R, \tilde{z}, \tilde{t}\right)=0 \\
\tilde{v}_{z}^{1}(\tilde{r}, \tilde{z}, 0)=0, \tilde{v}_{z}^{1}(\tilde{r}, 0, t)=\tilde{v}_{z}^{1}(\tilde{r}, L, t)=0
\end{gathered}
$$

we see that the axial momentum equation implies, together with the periodicity in $y$, that $\varphi=0$. Since $\alpha(\tilde{r}, \tilde{z}, \tilde{t})$ is zero at $\tilde{r}=1+\Xi \tilde{\eta}^{0} / R$ we conclude that $\tilde{\tilde{p}}^{2}=0$. Therefore, correction $\left(\tilde{v}_{z}^{1}, \tilde{v}_{r}^{1}\right),\left(\tilde{\tilde{p}}^{1}, \tilde{\tilde{p}}^{2}\right)=(0,0)$ is obtained by solving $(7.26)-(7.28)$ with $\varphi=\tilde{\tilde{p}}^{1}=0$. This way we have obtained a closed problem. Functions $\left(\tilde{v}_{z}^{0}+\varepsilon \tilde{v}_{z}^{1}, \varepsilon \tilde{v}_{r}^{1}\right)$ and $\tilde{\eta}^{0}$ (namely, $\tilde{\tilde{p}}^{0} / \varepsilon$ ) also satisfy problem (5.15)-(5.22) to $\varepsilon^{2}$-order. More precisely, since $\tilde{\tilde{p}}^{0}=\varepsilon \tilde{p}$ and due to the boundary conditions for the pressure, we have that $\tilde{\tilde{p}}^{0}$ is of order $\varepsilon$. Consequently, both $\tilde{v}_{z}^{0}$ and $\tilde{v}_{r}^{1}$ are of order $\varepsilon$.

Proposition 7.1. The velocity field $\left(\tilde{v}_{z}^{0}+\varepsilon \tilde{v}_{z}^{1}, \varepsilon \tilde{v}_{r}^{1}\right)$ and the pressure field $\frac{1}{\varepsilon} \tilde{\tilde{p}}^{0}$ satisfy equations (5.15)-(5.22) to $\mathcal{O}\left(\varepsilon^{2}\right)$.

Proof. The functions $\left(\tilde{v}_{z}^{0}+\varepsilon \tilde{v}_{z}^{1}\right)$ and $\frac{1}{\varepsilon} \tilde{\tilde{p}}^{0}$ satisfy conservation of momentum (5.15) and conservation of mass (5.16) to order $\mathcal{O}\left(\varepsilon^{3}\right)$ and $\mathcal{O}\left(\varepsilon^{2}\right)$ respectively:

$$
\begin{gathered}
S h \frac{\partial}{\partial \tilde{t}}\left(\tilde{v}_{z}^{0}+\varepsilon \tilde{v}_{z}^{1}\right)+\left(\tilde{v}_{z}^{0}+\varepsilon \tilde{v}_{z}^{1}\right) \frac{\partial}{\partial \tilde{z}}\left(\tilde{v}_{z}^{0}+\varepsilon \tilde{v}_{z}^{1}\right)+\tilde{v}_{\tilde{r}}^{1} \frac{\partial}{\partial \tilde{r}}\left(\tilde{v}_{z}^{0}+\varepsilon \tilde{v}_{z}^{1}\right)+\frac{1}{\varepsilon} \frac{\partial \tilde{\tilde{p}}}{\partial \tilde{z}} \\
-\frac{1}{R e} \Delta_{r}\left(\tilde{v}_{z}^{0}+\varepsilon \tilde{v}_{z}^{1}\right)=\varepsilon\left(\tilde{v}_{z}^{0} \frac{\partial \tilde{v}_{z}^{1}}{\partial \tilde{z}}+\tilde{v}_{z}^{1} \frac{\partial \tilde{v}_{z}^{0}}{\partial \tilde{z}}+\tilde{v}_{r}^{1} \frac{\partial \tilde{v}_{z}^{1}}{\partial \tilde{r}}\right)+\varepsilon^{2} \tilde{v}_{z}^{1} \frac{\partial \tilde{v}_{z}^{1}}{\partial \tilde{z}}=\mathcal{O}\left(\varepsilon^{3}\right) \\
\text { and } \frac{1}{\tilde{r}} \frac{\partial}{\partial \tilde{r}}\left(\tilde{r} \tilde{v}_{r}^{1}\right)+\frac{\partial}{\partial \tilde{z}}\left(\tilde{v}_{z}^{0}+\varepsilon \tilde{v}_{z}^{1}\right)=\varepsilon \frac{\partial \tilde{v}_{z}^{1}}{\partial \tilde{z}}=\mathcal{O}\left(\varepsilon^{2}\right) .
\end{gathered}
$$

In the pressure-radius relationship, ignoring the shear modulus term, and recalling that the relation between the pressure and the radial displacement was used up to order $\varepsilon^{2}$, we see that the functions $\left(\tilde{v}_{z}^{0}+\varepsilon \tilde{v}_{z}^{1}\right)$ and $\frac{1}{\varepsilon} \tilde{\tilde{p}}^{0}$ satisfy $(5.15)-(5.22)$ to $\mathcal{O}\left(\varepsilon^{2}\right)$.

We summarize the main steps in the derivation of the model and the final equations in dimensional form.

7.2.3. Summary: Dimensional Form of the Reduced Equations. The following is an $\varepsilon^{2}$-approximation of the 3-D axially symmetric flow of an incompressible, Newtonian fluid in an elastic tube described in $\S 2$ as Problem $\mathrm{P}^{\varepsilon}$. The unknown functions are the velocity $\left(v_{z}^{0}+v_{z}^{1}, v_{r}^{1}\right)$ and the radial displacement $\eta^{0}$. The pressure $p=p_{\text {ref }}+p^{0}$ is then recovered via the pressure-radius relationship (7.34). The radius 
of the deformed vessel at every time step is given by $r_{\text {vessel }}=R+\eta^{0}(z, t)$. The equations hold under the following

ASSUMPTIONS:

- The domain is axially symmetric with small aspect ratio $\epsilon=R / L<<1$.

- Longitudinal displacement is negligible.

- Radial displacement is not too large, i.e., $\Xi / R \leq \epsilon$.

- The base pressure loading of the elastic tube is zero and the coefficient of longitudinal tension at rest $k G$ is such that $k G\left(1-\sigma^{2}\right) / E$ is of order one.

- The initial tube radius is constant.

- The $S h$ number is not small, i.e., $S h>1$, and $R e$ is medium (several hundreds).

- The $z$-derivatives of non-dimensional quantities are not necessarily small, i.e., they are of order $O(1)$.

COUPLING AT THE LATERAL BOUNDARY:

- Continuity of velocity and contact forces is performed at the deformed interface.

- Fluid-structure contact force relationship is linear, see (5.13) and (5.14).

STEP 1.(The ZERO-TH ORDER APPROXIMATION) Look for $v_{z}^{0}=v_{z}^{0}(r, z, t)$ and $\eta^{0}=$ $\eta^{0}(z, t)$ and then recover $p^{0}=p^{0}(z, t)$ by solving the following free-boundary problem defined on the domain $0 \leq z \leq L, 0 \leq r \leq R+\eta^{0}(z, t)$

$$
\begin{gathered}
\frac{\partial\left(R+\eta^{0}\right)^{2}}{\partial t}+\frac{\partial}{\partial z} \int_{0}^{R+\eta^{0}} 2 r v_{z}^{0} d r=0, \\
\rho \frac{\partial v_{z}^{0}}{\partial t}+\frac{\partial}{\partial z}\left(\frac{h E}{R\left(1-\sigma^{2}\right)} \frac{\eta^{0}}{R+\eta^{0}}\right)=\mu \frac{1}{r} \frac{\partial}{\partial r}\left(r \frac{\partial v_{z}^{0}}{\partial r}\right), \\
v_{z}^{0}(0, z, t) \text { bounded, } v_{z}^{0}\left(R+\eta^{0}(z, t), z, t\right)=0 \text { and } v_{z}^{0}(r, z, 0)=0,
\end{gathered}
$$

with the following inlet and outlet boundary conditions

$$
\begin{aligned}
& p=P_{1}(t)+p_{\text {ref }} \quad \text { for } \quad z=0,0 \leq r \leq R \quad \text { and } \quad \forall t \in \mathbb{R}_{+}, \\
& p=P_{2}(t)+p_{\text {ref }} \quad \text { for } \quad z=L, 0 \leq r \leq R \quad \text { and } \quad \forall t \in \mathbb{R}_{+} .
\end{aligned}
$$

The pressure $p$ is linked to $\eta^{0}$ via

$$
p(z, t)=p_{\text {ref }}+\frac{h E}{R\left(1-\sigma^{2}\right)} \frac{\eta^{0}}{R} .
$$

STEP 2.(The $\varepsilon$-CORRECTION FOR THE VELOCITY) Solve for $v_{z}^{1}=v_{z}^{1}(r, z, t)$ and $v_{r}^{1}=v_{r}^{1}(r, z, t)$ by first recovering $v_{r}^{1}$ via

$$
r v_{r}^{1}(r, z, t)=\left(R+\eta^{0}\right) \frac{\partial \eta^{0}}{\partial t}+\int_{r}^{R+\eta^{0}} \frac{\partial v_{z}^{0}}{\partial z}(\xi, z, t) \xi d \xi
$$

and then solve the following linear fixed boundary problem for $v_{z}^{1}$, defined on the domain $0 \leq z \leq L, 0 \leq r \leq R+\eta^{0}(z, t)$ :

$$
\begin{gathered}
\frac{\partial v_{z}^{1}}{\partial t}-\nu \frac{1}{r} \frac{\partial}{\partial r}\left(r \frac{\partial v_{z}^{1}}{\partial r}\right)=-S_{v_{z}^{1}}(r, z, t) \\
v_{z}^{1}(0, z, t) \text { bounded, } v_{z}^{1}\left(R+\eta^{0}(z, t), z, t\right)=0 \\
v_{z}^{1}(r, 0, t)=v_{z}^{1}(r, L, t)=0 \quad \text { and } \quad v_{z}^{1}(r, z, 0)=0,
\end{gathered}
$$


where $S_{v_{z}^{1}}(r, z, t)$ contains the already calculated functions and is defined by

$$
S_{v_{z}^{1}}(r, z, t)=v_{r}^{1} \frac{\partial v_{z}^{0}}{\partial r}+v_{z}^{0} \frac{\partial v_{z}^{0}}{\partial z}
$$

Here $\nu=\mu / \rho$ is the kinematic viscosity coefficient. Notice that the boundary condition is evaluated at the deformed boundary whose $\varepsilon^{2}$-approximation is obtained in the previous step. In the next section we explicitly take into account the assumption that $\Xi / R \leq \epsilon$ and perform an asymptotic expansion with respect to the small displacement.

7.2.4. Expansion with respect to the radial displacement $\Xi / R \leq \epsilon$. Introduce the following expansions of the dependent variables $\left(w+\varepsilon \tilde{v}_{z}^{1}, \varepsilon \tilde{v}_{r}^{1}\right), \tilde{\eta}^{0}, \tilde{\tilde{p}}^{0}$ :

$$
\begin{gathered}
\tilde{\eta}^{0}=\tilde{\eta}^{0,0}+\frac{\Xi}{R} \tilde{\eta}^{0,1}+\ldots, \quad \tilde{\tilde{p}}^{0}=\tilde{\tilde{p}}^{0,0}+\frac{\Xi}{R} \tilde{\tilde{p}}^{0,1}+\ldots \\
w=w^{0}+\frac{\Xi}{R} w^{1}+\ldots, \quad \tilde{v}_{z}^{1}=\tilde{v}_{z}^{1,0}+\ldots, \quad \tilde{v}_{r}^{1}=\tilde{v}_{r}^{1,0}+\ldots
\end{gathered}
$$

Plug these expansions into (7.14)-(7.15) and obtain the following equations of order zero and one, respectively:

$$
\begin{aligned}
& S h_{0} \frac{\partial w^{0}}{\partial \tilde{t}}-\frac{1}{R e_{0}} \frac{1}{\tilde{r}} \frac{\partial}{\partial \tilde{r}}\left(\tilde{r} \frac{\partial w^{0}}{\partial \tilde{r}}\right)=-\frac{\partial \tilde{\tilde{p}}^{0,0}}{\partial \tilde{z}}(\tilde{z}, \tilde{t})=-\left(\frac{E h}{\mathcal{P} L\left(1-\sigma^{2}\right)}\right) \frac{\partial \tilde{\eta}^{0,0}}{\partial \tilde{z}} \\
& w^{0}(0, \tilde{z}, \tilde{t}) \text { bounded, } \quad w^{0}(1, \tilde{z}, \tilde{t})=0 \quad \text { and } \quad w^{0}(\tilde{r}, \tilde{z}, 0)=0,
\end{aligned}
$$

and

$$
\begin{aligned}
& S h_{0} \frac{\partial w^{1}}{\partial \tilde{t}}-\frac{1}{R e_{0}} \frac{1}{\tilde{r}} \frac{\partial}{\partial \tilde{r}}\left(\tilde{r} \frac{\partial w^{1}}{\partial \tilde{r}}\right)=-\frac{\partial \tilde{\tilde{p}}^{0,1}}{\partial \tilde{z}}(\tilde{z}, \tilde{t})=-\left(\frac{E h}{\mathcal{P} L\left(1-\sigma^{2}\right)}\right) \frac{\partial \tilde{\eta}^{0,1}}{\partial \tilde{z}}, \\
& w^{1}(0, \tilde{z}, \tilde{t}) \text { bounded, } \quad w^{1}(1, \tilde{z}, \tilde{t})=-\tilde{\eta}^{0,0} \frac{\partial w^{0}}{\partial \tilde{r}}(1, \tilde{z}, \tilde{t}) \text { and } w^{1}(\tilde{r}, \tilde{z}, 0)=0,
\end{aligned}
$$

where we have linearized the lateral boundary condition. Note that $\tilde{\eta}^{0,0} \frac{\partial w^{0}}{\partial \tilde{r}}(1, \tilde{z}, \tilde{t})=0$ at $\tilde{t}=0$. Both of these problems can be solved efficiently by using the auxiliary (homogeneous) problem

$$
\begin{aligned}
& \frac{\partial \tilde{\zeta}}{\partial \tilde{t}}-\frac{1}{\tilde{r}} \frac{\partial}{\partial \tilde{r}}\left(\tilde{r} \frac{\partial \tilde{\zeta}}{\partial \tilde{r}}\right)=0 \quad \text { in }(0,1) \times(0, \infty) \\
& \tilde{\zeta}(0, \tilde{t}) \text { is bounded }, \quad \tilde{\zeta}(1, \tilde{t})=0 \text { and } \tilde{\zeta}(\tilde{r}, 0)=1 .
\end{aligned}
$$

Then, by linear parabolic theory, $\tilde{\zeta}$ decays in time exponentially, with the rate equal to the first zero of the Bessel function $J_{0}$. $\tilde{\zeta}$ is non-negative by the maximum principle. We set

$$
\tilde{\mathcal{K}}(t)=2 \int_{0}^{1} \tilde{\zeta}(\tilde{r}, \tilde{t}) \tilde{r} d \tilde{r} .
$$

We now solve equations (7.35)-(7.36). The following operators will be useful. Let $f=f(\tilde{z}, \tilde{t})$. Define $(\tilde{\zeta} \star f)(\tilde{r}, \tilde{z}, \tilde{t})$ and $(\tilde{\mathcal{K}} \star f)(\tilde{z}, \tilde{t})$ to be the following integral 
operators with the kernels $\tilde{\zeta}$ and $\tilde{\mathcal{K}}$ respectively

$$
\begin{aligned}
(\tilde{\zeta} \star f)(\tilde{r}, \tilde{z}, \tilde{t}) & :=\int_{0}^{\tilde{t}} \tilde{\zeta}\left(\tilde{r}, \frac{\tilde{t}-\tau}{S h_{0} R e_{0}}\right) f(\tilde{z}, \tau) d \tau, \\
(\tilde{\mathcal{K}} \star f)(\tilde{z}, \tilde{t}) & :=\int_{0}^{\tilde{t}} \tilde{\mathcal{K}}\left(\frac{\tilde{t}-\tau}{S h_{0} R e_{0}}\right) f(\tilde{z}, \tau) d \tau .
\end{aligned}
$$

Then, the solution of (7.35) in terms of $\frac{\partial \tilde{\tilde{p}}^{0,0}}{\partial \tilde{z}}$ can be written as

$$
w^{0}(\tilde{r}, \tilde{z}, \tilde{t})=-\frac{1}{S h_{0}}\left(\tilde{\zeta} \star \frac{\partial \tilde{\tilde{p}}^{0,0}}{\partial \tilde{z}}\right)(\tilde{r}, \tilde{z}, \tilde{t}) .
$$

Using

$$
\frac{\partial \tilde{\tilde{p}}^{0,0}}{\partial \tilde{z}}=\left(\frac{E h}{\mathcal{P} L\left(1-\sigma^{2}\right)}\right) \frac{\partial \tilde{\eta}^{0,0}}{\partial \tilde{z}}
$$

and (7.39) in (7.10) we get the following equation for the first term of the radial displacement, $\tilde{\eta}^{0,0}$, at the zero-th order, holding in $(0, L) \times(0, \infty)$

$$
2 S h_{0} \frac{\partial \tilde{\eta}^{0,0}}{\partial \tilde{t}}(\tilde{z}, \tilde{t})-\frac{h E}{L \mathcal{P}\left(1-\sigma^{2}\right)} \frac{\partial}{\partial \tilde{z}}\left(\tilde{\mathcal{K}} \star \frac{\partial \tilde{\eta}^{0,0}}{\partial \tilde{z}}\right)(\tilde{z}, \tilde{t})=0 .
$$

For the explicit formula for the Laplace transform of $\tilde{\eta}^{0,0}$ see Appendix A. Next we calculate $\tilde{\eta}^{0,1}$ by first expressing $w^{1}$. Integrate (7.36) and take the linearized boundary condition in (7.36) into account to obtain

$$
\begin{aligned}
w^{1}(\tilde{r}, \tilde{z}, \tilde{t}) & =-\tilde{\eta}^{0,0} \frac{\partial w^{0}}{\partial \tilde{r}}(1, \tilde{z}, \tilde{t})-\frac{1}{S h_{0}}\left(\tilde{\zeta} \star \frac{\partial \tilde{\tilde{p}}^{0,1}}{\partial \tilde{z}}\right)(\tilde{r}, \tilde{z}, \tilde{t}) \\
& +\left(\tilde{\zeta} \star \frac{\partial}{\partial \tilde{t}}\left[\left.\tilde{\eta}^{0,0} \frac{\partial w^{0}}{\partial \tilde{r}}\right|_{\tilde{r}=1}\right]\right)(\tilde{r}, \tilde{z}, \tilde{t}) .
\end{aligned}
$$

Here, the last expression means

$$
\left(\tilde{\zeta} \star \frac{\partial}{\partial \tilde{t}}\left[\left.\tilde{\eta}^{0,0} \frac{\partial w^{0}}{\partial \tilde{r}}\right|_{\tilde{r}=1}\right]\right)(\tilde{r}, \tilde{z}, \tilde{t})=\int_{0}^{\tilde{t}} \tilde{\zeta}\left(\tilde{r}, \frac{\tilde{t}-\tau}{S h_{0} R e_{0}}\right) \frac{\partial}{\partial \tau}\left(\tilde{\eta}^{0,0}(\tilde{z}, \tau) \frac{\partial w^{0}}{\partial \tilde{r}}(1, \tilde{z}, \tau)\right) d \tau
$$

From here we get the integral of $w^{1}$ in terms of the kernel $\tilde{\mathcal{K}}$

$$
\begin{gathered}
2 \int_{0}^{1} w^{1}(\tilde{r}, \tilde{z}, \tilde{t}) \tilde{r} d \tilde{r}=-\tilde{\eta}^{0,0} \frac{\partial w^{0}}{\partial \tilde{r}}(1, \tilde{z}, \tilde{t})-\frac{1}{S h_{0}}\left(\tilde{\mathcal{K}} \star \frac{\partial \tilde{\tilde{p}}^{0,1}}{\partial \tilde{z}}\right)(\tilde{z}, \tilde{t}) \\
+\left(\tilde{\mathcal{K}} \star \frac{\partial}{\partial \tilde{t}}\left[\left.\tilde{\eta}^{0,0} \frac{\partial w^{0}}{\partial \tilde{r}}\right|_{\tilde{r}=1}\right]\right)(\tilde{z}, \tilde{t}) .
\end{gathered}
$$

Plugging (7.43) into (7.10) we obtain the equation for $\tilde{\eta}^{0,1}$ in $(0, L) \times(0, \infty)$

$$
2 S h_{0} \frac{\partial \tilde{\eta}^{0,1}}{\partial \tilde{t}}-\frac{h E}{L \mathcal{P}\left(1-\sigma^{2}\right)} \frac{\partial}{\partial \tilde{z}}\left(\tilde{\mathcal{K}} \star \frac{\partial \tilde{\eta}^{0,1}}{\partial \tilde{z}}\right)(\tilde{z}, \tilde{t})=-S_{\tilde{\eta}^{0,1}}(\tilde{z}, \tilde{t})
$$


where

$$
\begin{gathered}
S_{\tilde{\eta}^{0,1}}(\tilde{z}, \tilde{t}):=2 S h_{0} \eta^{0,0} \frac{\partial \eta^{0,0}}{\partial \tilde{t}}-S h_{0} \frac{\partial}{\partial \tilde{z}}\left(\tilde{\eta}^{0,0} \frac{\partial w^{0}}{\partial \tilde{r}}(1, \tilde{z}, \tilde{t})\right) \\
+S h_{0} \frac{\partial}{\partial \tilde{z}}\left(\tilde{\mathcal{K}} \star \frac{\partial}{\partial \tilde{t}}\left[\left.\tilde{\eta}^{0,0} \frac{\partial w^{0}}{\partial \tilde{r}}\right|_{\tilde{r}=1}\right]\right)(\tilde{z}, \tilde{t}) .
\end{gathered}
$$

We perform the same expansions for the correction of order $\varepsilon$. Equation (7.25) implies the following zero-order approximation of $\tilde{v}_{r}^{1,0}$ with respect to $\frac{\Xi}{R}$ :

$$
\tilde{r} w^{0}(\tilde{r}, \tilde{z}, \tilde{t})=\frac{\partial \tilde{\eta}^{0,0}}{\partial \tilde{t}}+\int_{\tilde{r}}^{1} \frac{\partial w^{0}}{\partial \tilde{z}}(\xi, \tilde{z}, \tilde{t}) \xi d \xi
$$

Equations (7.26)-(7.28) imply, after taking into account $\varphi=\tilde{\tilde{p}}^{1}=0$, the following zero-order equation for $\tilde{v}_{z}^{1,0}$ :

$$
\begin{gathered}
S h_{0} \frac{\partial \tilde{v}_{z}^{1,0}}{\partial \tilde{t}}-\frac{1}{R e_{0}} \frac{1}{\tilde{r}} \frac{\partial}{\partial \tilde{r}}\left(\tilde{r} \frac{\partial \tilde{v}_{z}^{1,0}}{\partial \tilde{r}}\right)=-S_{\tilde{v}_{z}^{1,0}}(\tilde{r}, \tilde{z}, \tilde{t}), \\
\tilde{v}_{z}^{1,0}(0, \tilde{z}, \tilde{t}) \text { bounded, } \tilde{v}_{z}^{1,0}(1, \tilde{z}, \tilde{t})=0 \quad \text { and } \quad \tilde{v}_{z}^{1,0}(\tilde{r}, \tilde{z}, 0)=0,
\end{gathered}
$$

where

$$
S_{\tilde{v}_{z}^{1,0}}(\tilde{r}, \tilde{z}, \tilde{t})=\tilde{v}_{r}^{1,0} \frac{\partial w^{0}}{\partial \tilde{r}}+w^{0} \frac{\partial w^{0}}{\partial \tilde{z}} .
$$

With the calculations presented in this section we have derived a set of linear equations that are an $\varepsilon^{2}$-approximation of Problem $\mathrm{P}^{\varepsilon}$ when $\Xi / R \leq \varepsilon$. First one recovers $\tilde{\eta}^{0,0}$ by solving (7.41) with the appropriate initial and boundary conditions. Next, the zero-th approximation of the unidirectional velocity $w^{0}$ is recovered by calculating (7.39). Pressure $\tilde{\tilde{p}}^{0,0}$ follows from (7.40). The 2nd-order correction $\tilde{\eta}^{0,1}$ for the displacement is obtained by solving (7.44) with appropriate initial and boundary conditions. This correction is necessary to recover the next term in the approximation of the unidirectional flow $w^{1}$ via (7.2.4). Finally, the $\varepsilon$-correction for the unidirectional velocity is obtained by recovering $\tilde{v}_{z}^{1,0}$ and $\tilde{v}_{r}^{1,0}$ via (7.39) and (7.45) respectively. Notice that since the radius of the vessel in non-dimensional variables reads

$1+\frac{\Xi}{R} \tilde{\eta}=1+\frac{\Xi}{R}\left(\tilde{\eta}^{0}+\varepsilon \tilde{\eta}^{1}+\cdots\right)=1+\frac{\Xi}{R} \tilde{\eta}=1+\frac{\Xi}{R}\left(\left(\tilde{\eta}^{0,0}+\frac{\Xi}{R} \tilde{\eta}^{0,1}+\cdots\right)+\varepsilon \tilde{\eta}^{1}+\cdots\right)$,

assumption $\frac{\Xi}{R} \leq \varepsilon$ implies that the $\varepsilon^{2}$ approximation of the solution is achieved already with the $\tilde{\eta}^{0,0}$ term. However, we need to calculate $\tilde{\eta}^{0,1}$ in order to recover the $\varepsilon^{2}$ approximation of the velocity. A similar argument holds for the calculation of the scaled pressure $\tilde{\tilde{p}}$. We summarize the main steps written in dimensional form, in the following section. We will be utilizing the following notation (relationship) between the non-dimensional and dimensional variables:

$$
\begin{aligned}
\eta= & \eta^{0,0}+\eta^{0,1}+\cdots=\Xi\left(\tilde{\eta}^{0,0}+\frac{\Xi}{R} \tilde{\eta}^{0,1}+\cdots\right), \text { where } \\
& \eta^{0,0}=\Xi \tilde{\eta}^{0,0}, \eta^{0,1}=\Xi \bar{\Xi} \tilde{\eta}^{0,1} \\
v_{z}= & v_{z}^{0,0}+v_{z}^{0,1}+v_{z}^{1,0}+\cdots=V\left(\tilde{v}_{z}^{0,0}+\frac{\Xi}{R} \tilde{v}_{z}^{0,1}+\varepsilon \tilde{v}_{z}^{1,0}+\cdots\right), \text { where } \\
& v_{z}^{0,0}=V \tilde{v}_{z}^{0,0}, v_{z}^{0,1}=V \frac{\Xi}{R} \tilde{v}_{z}^{0,1}, v_{z}^{1,0}=V \varepsilon \tilde{v}_{z}^{1,0}, \\
v_{r}= & v_{r}^{1,0}+\cdots=V\left(\varepsilon \tilde{v}_{r}^{1,0}+\cdots\right), \text { where } v_{r}^{1,0}=V \varepsilon \tilde{v}_{r}^{1,0} .
\end{aligned}
$$


7.2.5. Summary: Expansion with respect to the radial displacement: equations in dimensional form. Find a solution consisting of: (1) the velocity field $\left(\left(v_{z}^{0,0}+v_{z}^{0,1}\right)+v_{z}^{1,0}, v_{r}^{1,0}\right)$ where $\left(v_{z}^{0,0}+v_{z}^{0,1}, 0\right)$ is the unidirectional velocity and $\left(v_{z}^{1,0}, v_{r}^{1,0}\right)$ is its $\varepsilon$-correction, (2) the radial displacement $\eta^{0,0}$ and (3) the leading-order pressure $p=p_{\text {ref }}+p^{0,0}$.

STEP 1. (The ZERoth order Approximation) Find $v_{z}^{0,0}(r, z, t), \eta^{0,0}(z, t)$ and $p^{0,0}(z, t)$ such that

$$
\begin{gathered}
\frac{\partial\left(\eta^{0,0}\right)}{\partial t}+\frac{1}{R} \frac{\partial}{\partial z} \int_{0}^{R} r v_{z}^{0,0} d r=0 \\
\rho \frac{\partial v_{z}^{0,0}}{\partial t}-\mu \frac{1}{r} \frac{\partial}{\partial r}\left(r \frac{\partial v_{z}^{0,0}}{\partial r}\right)=-\frac{\partial p^{0,0}}{\partial z}(z, t), \quad \frac{\partial p^{0,0}}{\partial z}(z, t)=\left(\frac{E h}{R^{2}\left(1-\sigma^{2}\right)}\right) \frac{\partial \eta^{0,0}}{\partial z} \\
\text { with } \quad v_{z}^{0,0}(0, z, t) \text { bounded, } \quad v_{z}^{0,0}(R, z, t)=0 \\
p^{0,0}(z, 0)=p_{\text {ref }}, \quad \eta^{0,0}(z, 0)=v_{z}^{0,0}(r, z, 0)=0 \\
\eta^{0,0}(0, t)=\frac{R^{2}\left(1-\sigma^{2}\right)}{E h} P_{1}(t), \quad \eta^{0,0}(L, t)=\frac{R^{2}\left(1-\sigma^{2}\right)}{E h} P_{2}(t) .
\end{gathered}
$$

Then recover the $\Xi / R$-correction $v_{z}^{0,1}(r, z, t), \eta^{0,1}(z, t)$ and $p^{0,1}(z, t)$ by solving

$$
\begin{gathered}
\frac{\partial\left(\eta^{0,1}\right)}{\partial t}+\frac{1}{R} \frac{\partial}{\partial z} \int_{0}^{R} r v_{z}^{0,1} d r=-\frac{1}{R} \eta^{0,0} \frac{\partial \eta^{0,0}}{\partial t} \\
\rho \frac{\partial v_{z}^{0,1}}{\partial t}-\mu \frac{1}{r} \frac{\partial}{\partial r}\left(r \frac{\partial v_{z}^{0,1}}{\partial r}\right)=-\frac{\partial p^{0,1}}{\partial z}(z, t), \quad \frac{\partial p^{0,1}}{\partial z}(z, t)=\left(\frac{E h}{R^{2}\left(1-\sigma^{2}\right)}\right) \frac{\partial \eta^{0,1}}{\partial z}
\end{gathered}
$$

with

$$
\begin{gathered}
v_{z}^{0,1}(0, z, t) \text { bounded, } \quad v_{z}^{0,1}(R, z, t)=-\eta^{0,0} \frac{\partial v_{z}^{0,0}}{\partial r}(R, z, t), \\
p^{0,1}(z, 0)=0, \quad \eta^{0,1}(z, 0)=v_{z}^{0,1}(r, z, 0)=0 \\
\eta^{0,1}(0, t)=\eta^{0,1}(L, t)=0 .
\end{gathered}
$$

We can solve these problems efficiently by considering the auxiliary problem

$$
\left\{\begin{array}{l}
\frac{\partial \zeta}{\partial t}-\frac{1}{r} \frac{\partial}{\partial r}\left(r \frac{\partial \zeta}{\partial r}\right)=0 \quad \text { in }(0, R) \times(0, \infty) \\
\zeta(0, t) \text { is bounded }, \quad \zeta(R, t)=0 \text { and } \zeta(r, 0)=1,
\end{array}\right.
$$

and the mean of $\zeta$ in the radial direction

$$
\mathcal{K}(t)=2 \int_{0}^{R} \zeta(r, t) r d r
$$

which can both be evaluated in terms of the Bessel's functions. The unidirectional solution can then be written in terms of the following operators

$$
\begin{aligned}
(\zeta \star f)(r, z, t) & :=\int_{0}^{t} \zeta\left(r, \frac{\mu(t-\tau)}{\rho}\right) f(z, \tau) d \tau, \\
(\mathcal{K} \star f)(z, t) & :=\int_{0}^{t} \mathcal{K}\left(\frac{\mu(t-\tau)}{\rho}\right) f(z, \tau) d \tau .
\end{aligned}
$$


We write the explicit solution strategy below. Denote $C=E h /\left(R^{2}\left(1-\sigma^{2}\right)\right)$.

STEP 1. REVISITED. (ExPlicit Solution Method)

- STEP 1a. Find $\eta^{0,0}, p^{0,0}, v_{z}^{0,0}$ by solving the following initial-boundary value problem of Biot type with memory:

$$
\left\{\begin{array}{l}
\frac{\partial \eta^{0,0}}{\partial t}(z, t)=\frac{C}{2 \rho R} \frac{\partial^{2}\left(\mathcal{K} \star \eta^{0,0}\right)}{\partial z^{2}}(z, t) \text { on }(0, L) \times(0,+\infty) \\
\eta^{0,0}(0, t)=P_{1}(t) / C, \quad \eta^{0,0}(L, t)=P_{2}(t) / C \quad \text { and } \quad \tilde{\eta}^{0,0}(z, 0)=0 .
\end{array}\right.
$$

Recover $\quad \frac{\partial p^{0,0}}{\partial z}(z, t)=C \frac{\partial \eta^{0,0}}{\partial z}(z, t)$.

Calculate $v_{z}^{0,0}$ by solving

$$
\left\{\begin{array}{l}
\rho \frac{\partial v_{z}^{0,0}}{\partial t}-\mu \frac{1}{r} \frac{\partial}{\partial r}\left(r \frac{\partial v_{z}^{0,0}}{\partial r}\right)=-\frac{\partial p^{0,0}}{\partial z}(z, t) \\
v_{z}^{0,0}(0, z, t) \text { bounded, } v_{z}^{0,0}(R, z, t)=0
\end{array}\right.
$$

Explicit solution of (7.49) is: $v_{z}^{0,0}(r, z, t)=-\frac{1}{\rho}\left(\zeta \star \frac{\partial p^{0,0}}{\partial z}\right)(r, z, t)$.

- STEP 1b. Find $\eta^{0,1}, p^{0,1}, v_{z}^{0,1}$ by solving the following initial-boundary value problem on $(0, L) \times(0,+\infty)$ with memory:

$$
\left\{\begin{array}{l}
\frac{\partial \eta^{0,1}}{\partial t}(z, t)=\frac{C}{2 \rho R} \frac{\partial^{2}\left(\mathcal{K} \star \eta^{0,1}\right)}{\partial z^{2}}(z, t)-S_{\eta^{0,1}}(z, t) \\
\eta^{0,1}(0, t)=\eta^{0,1}(L, t)=0 \text { and } \eta^{0,1}(z, 0)=0
\end{array}\right.
$$

where

$$
\begin{aligned}
S_{\eta^{0,1}}(z, t):= & \frac{1}{R} \eta^{0,0} \frac{\partial \eta^{0,0}}{\partial t}-\frac{R}{2} \frac{\partial}{\partial z}\left(\left.\eta^{0,0} \frac{\partial v_{z}^{0,0}}{\partial r}\right|_{r=R}\right) \\
& +\frac{1}{2 R} \frac{\partial}{\partial z}\left(\mathcal{K} \star \frac{\partial}{\partial t}\left(\left.\eta^{0,0} \frac{\partial v_{z}^{0,0}}{\partial r}\right|_{r=R}\right)\right) .
\end{aligned}
$$

Recover $\frac{\partial p^{0,1}}{\partial z}(z, t)=C \frac{\partial \eta^{0,1}}{\partial z}(z, t)$.

Calculate $v_{z}^{0,1}$ by solving

$$
\left\{\begin{array}{l}
\rho \frac{\partial v_{z}^{0,1}}{\partial t}-\mu \frac{1}{r} \frac{\partial}{\partial r}\left(r \frac{\partial v_{z}^{0,1}}{\partial r}\right)=-\frac{\partial p^{0,1}}{\partial z}(z, t) \\
v_{z}^{0,1}(0, z, t) \text { bounded, } \quad v_{z}^{0,1}(R, z, t)=-\eta^{0,0} \frac{\partial v_{z}^{0,0}}{\partial r}(R, z, t) .
\end{array}\right.
$$

Explicit solution of (7.51) is:

$$
\begin{aligned}
v_{z}^{0,1}(r, z, t)= & -\eta^{0,0} \frac{\partial v_{z}^{0,0}}{\partial r}(R, z, t)-\frac{1}{\rho}\left(\zeta \star \frac{\partial p^{0,1}}{\partial z}\right)(r, z, t) \\
& +\left(\zeta \star \frac{\partial}{\partial t}\left[\left.\eta^{0,0} \frac{\partial v_{z}^{0,0}}{\partial r}\right|_{r=R}\right]\right)(r, z, t) .
\end{aligned}
$$

This way we have recovered the unidirectional velocity $\left(v_{z}^{0,0}+v_{z}^{0,1}, 0\right)$, the $\varepsilon^{2}$-approximation of the radial displacement $\eta^{0,0}$ and the $\varepsilon^{2}$-approximation of the pressure

$$
p(z, t)=p_{\text {ref }}+\frac{h E}{R\left(1-\sigma^{2}\right)} \frac{\eta^{0,0}(z, t)}{R} .
$$


STEP 2.(The $\varepsilon$-CORRECTION FOR The VELOCITY) Solve for $v_{z}^{1,0}=v_{z}^{1,0}(r, z, t)$ and $v_{r}^{1,0}=v_{r}^{1,0}(r, z, t)$ by first recovering $v_{r}^{1,0}$ via

$$
r v_{r}^{1,0}(r, z, t)=R \frac{\partial \eta^{0,0}}{\partial t}+\int_{r}^{R} \frac{\partial v_{z}^{0,0}}{\partial z}(\xi, z, t) \xi d \xi
$$

and then solve the following linear problem for $v_{z}^{1,0}$ defined on $(0, R) \times(0, L) \times(0, \infty)$

$$
\left\{\begin{array}{l}
\frac{\partial v_{z}^{1,0}}{\partial t}-\nu \frac{1}{r} \frac{\partial}{\partial r}\left(r \frac{\partial v_{z}^{1,0}}{\partial r}\right)=-S_{v_{z}^{1,0}}(r, z, t) \\
v_{z}^{1,0}(0, z, t) \text { bounded, } v_{z}^{1,0}(R, z, t)=0 \\
v_{z}^{1,0}(r, 0, t)=v_{z}^{1,0}(r, L, t)=0 \text { and } v_{z}^{1,0}(r, z, 0)=0
\end{array}\right.
$$

where $S_{v_{z}^{1,0}}(r, z, t)$ contains the already calculated functions and is defined by

$$
S_{v_{z}^{1,0}}(r, z, t)=v_{r}^{1,0} \frac{\partial v_{z}^{0,0}}{\partial r}+v_{z}^{0,0} \frac{\partial v_{z}^{0,0}}{\partial z} .
$$

We have completed an algorithm for the calculation of an $\varepsilon^{2}$-approximation of the solution to Problem $\mathrm{P}^{\varepsilon}$ in the case when $\Xi / R \leq \varepsilon$. The solution consists of the velocity $\left(v_{z}^{0,0}+v_{z}^{0,1}+v_{z}^{1,0}, v_{r}^{1,0}\right)$, the radial displacement $\eta^{0,0}$ and the pressure $p$ given via $(7.52)$.

8. Numerical Solution. We designed a numerical algorithm which is a combination of a finite difference method (FDM) and a finite element method (FEM). The finite difference method is used to solve for wave propagation in the elastic structure. The finite element method is used to solve for the velocity of the fluid. The algorithm is summarized below.

\section{THE NUMERICAL SCHEME}

1. Solve the auxiliary problem (7.46) using 1D FEM.

2. Compute $\mathcal{K}$ defined in (7.47) using numerical integration.

3. Approximation 0,0:

(a) Solve (7.48) for $\eta^{0,0}$ using implicit FDM.

(b) Solve (7.49) for $v_{z}^{0,0}$ using $1 \mathrm{D}$ FEM.

4. Approximation 0,1 :

(a) Solve (7.50) for $\eta^{0,1}$ using implicit FDM.

(b) Solve (7.51) for $v_{z}^{0,1}$ using 1D FEM.

5. Approximation 1,0:

(a) Solve (7.53) for $v_{r}^{1,0}$ using numerical integration.

(b) Solve (7.54) for $v_{z}^{1,0}$ using 1D FEM.

6. Compute the total approximation:

$$
v_{r}=v_{r}^{1,0}, v_{z}=v_{z}^{0,0}+v_{z}^{0,1}+v_{z}^{1,0}, \eta=\eta^{0,0}+\eta^{0,1} .
$$

First note that the problems for $\zeta, v_{z}^{0,0}, v_{z}^{0,1}$ and $v_{z}^{1,0}$ are all of the same form so the mass and stiffness matrices in the FEM method are equal, up to the boundary condition. Therefore they are generated only once. The same argument holds for the FDM used to solve for $\eta^{0,0}$ and $\eta^{0,1}$. The most time consuming is the calculation of the time integral appearing in the problems for $\eta^{0,0}$ and $\eta^{0,1}$. Nevertheless, the entire calculation lasts about one minute per cardiac cycle on a PC. This is because our algorithm consists of solving a sequence of $1 \mathrm{D}$ problems, so its complexity is the same as that of solving a 1D problem. The numerical simulation shown in Figure 8.1 

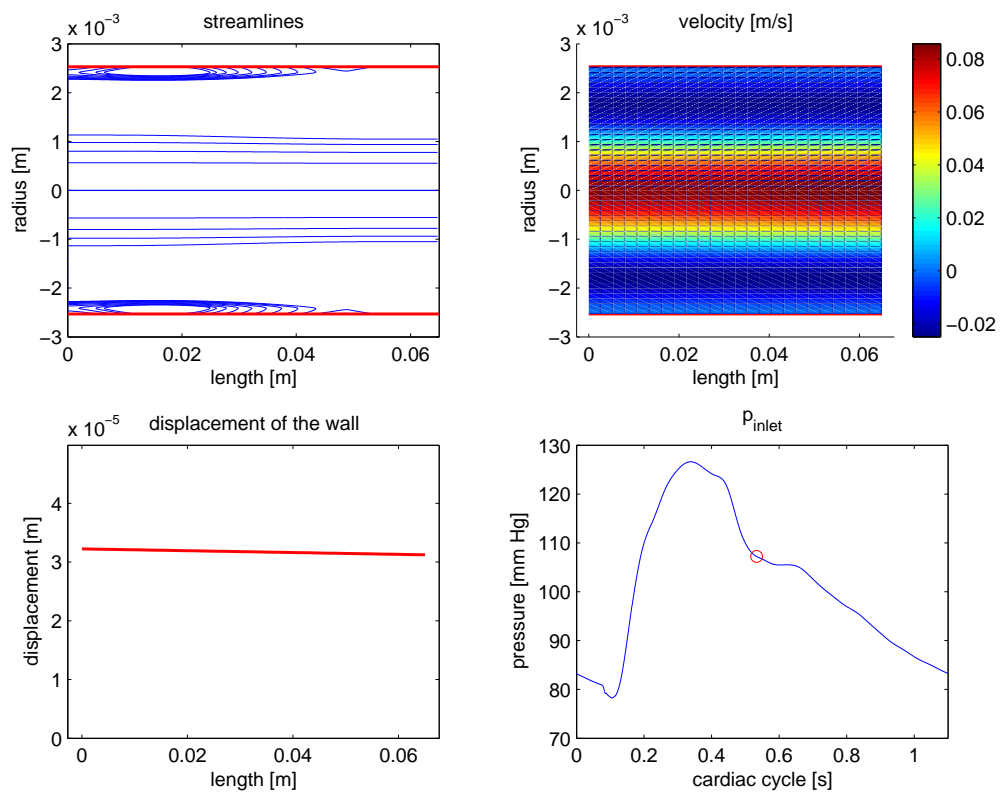

FIG. 8.1. Numerical simulation of flow through a linearly elastic tube with radius $R=0.0025 \mathrm{~m}$, length $L=0.065 \mathrm{~m}$, Young's modulus $E=400000 \mathrm{~Pa}$ and $h=0.0018 \mathrm{~m}$.

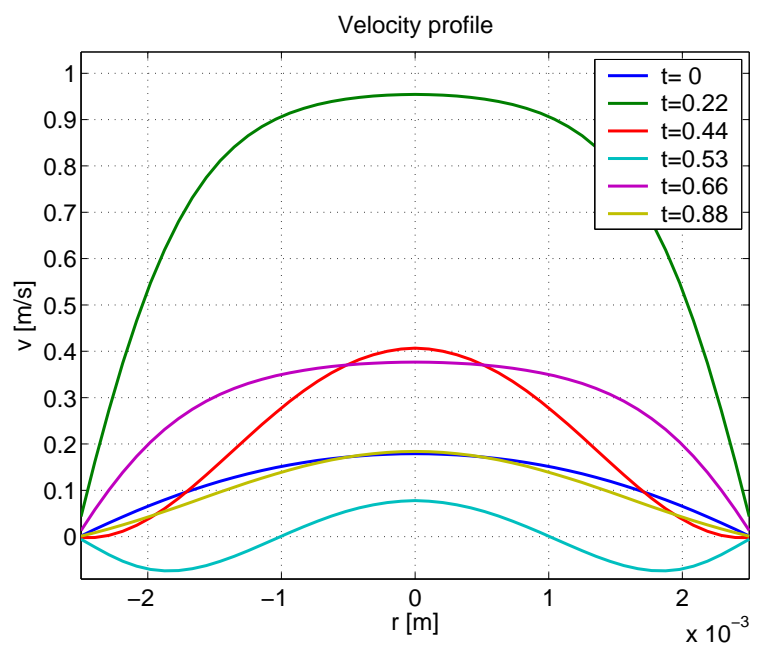

FIG. 8.2. Axial velocity profile at different times in one cardiac cycle.

indicates that our model captures two-dimensional flow phenomena. Figure 8.1 shows the streamlines, the speed and the vessel wall displacement taken in the middle of one cardiac cycle. The exact position in the cardiac cycle is shown with a red circle on the bottom-right graph of the inlet pressure wave as a function of time. The streamlines show recirculation of flow occurring briefly at this particular moment. The analysis presented in this paper implies that the solution approximates the original threedimensional problem to $\epsilon^{2}$-order. In Figure 8.2 we see the corresponding axial velocity profiles. As expected, the velocity profiles are of Womersley flow-type, calculated for 
compliant tubes. The brief recirculation zone shown in Figure 8.1 corresponds to the brief occurrence of negative axial velocity near the wall, shown in Figure 8.2 for $t=0.53$.

9. Final Remarks. We conclude by a couple of remarks related to the validity of the models. Our approximations are expected to work not only at moderate but also at high but laminar Reynolds numbers. In the turbulent flow regime, however, our approach in not likely to work and it should be modified. Also, taking the limit as $R e \rightarrow \infty$ in our analysis, even if formally possible, would very likely lead to wrong models. The reader interested in such flow regimes can look at [2] where the "Euler" variant of system (5.15)-(5.18) was studied, corresponding to $1 / R e=0$, with no-slip boundary conditions and with a convex velocity profile.

Appendix A. Explicit Laplace transform solution. In this Appendix we calculate the Laplace transform of the zero-th order approximation of the displacement when linear coupling is considered. We apply the Laplace transform to the auxiliary problem (7.37). The Laplace transform $\tilde{\tilde{\zeta}}$ of $\tilde{\zeta}$ satisfies

$$
\begin{gathered}
p \hat{\tilde{\zeta}}(p, \tilde{r})-\frac{1}{\tilde{r}} \frac{\partial}{\partial \tilde{r}}\left(\tilde{r} \frac{\partial \hat{\tilde{\zeta}}}{\partial \tilde{r}}(p, \tilde{r})\right)=1 \quad \text { on } \quad(0,1) \\
\hat{\tilde{\zeta}}(p, 0) \quad \text { is bounded, } \quad \hat{\tilde{\zeta}}(p, 1)=0 .
\end{gathered}
$$

This problem has a unique solution for all $p>0$ and the solution is given by

$$
\hat{\tilde{\zeta}}(p, \tilde{r})=\frac{1}{p}\left\{1-\frac{J_{0}(i \sqrt{p} \tilde{r})}{J_{0}(i \sqrt{p})}\right\}=\frac{1}{p}\left\{1-\frac{I_{0}(\sqrt{p} \tilde{r})}{I_{0}(\sqrt{p})}\right\}
$$

where $J_{0}$ is the Bessel function of order zero and $I_{0}$ is the modified Bessel function of order zero. The Laplace transform $\hat{\tilde{\mathcal{K}}}$ of the convolution kernel $\tilde{\mathcal{K}}$ is

$$
\begin{gathered}
\hat{\tilde{\mathcal{K}}}(p)=2 \int_{0}^{1} \hat{\tilde{\zeta}}(0, \tilde{r}) \tilde{r} d \tilde{r}=\frac{2}{p}\left\{\frac{1}{2}-\frac{1}{I_{0}(\sqrt{p})} \int_{0}^{1} \tilde{r} I_{0}(\sqrt{p} \tilde{r}) d \tilde{r}\right\} \\
=\frac{2}{p}\left\{\frac{1}{2}-\frac{1}{\sqrt{p}} \frac{I_{1}(\sqrt{p})}{I_{0}(\sqrt{p}}\right\},
\end{gathered}
$$

where $I_{1}(x)=-i J_{1}(i x)$ is the modified Bessel function of order 1 . We use this to explicitly calculate the Laplace transform of the solutions to the evolution problems (7.41) and (7.44) for $\tilde{\eta}^{0,0}$ and $\tilde{\eta}^{0,1}$. The homogeneous problems for both $\tilde{\eta}^{0,0}$ and $\tilde{\eta}^{0,1}$ have the form

$$
\begin{gathered}
S h_{0} \frac{\partial \tilde{\eta}}{\partial \tilde{t}}-\beta_{0} \frac{\partial^{2}}{\partial \tilde{z}^{2}}\left\{\tilde{\mathcal{K}}\left(\frac{\cdot}{R e_{0} S h_{0}}\right) \star \tilde{\eta}\right\}=0, \quad \text { in } \quad(0,1) \times \mathbb{R}^{+}, \\
\tilde{\eta}(0, \tilde{z})=0, \quad \tilde{\eta}(\tilde{t}, 0)=\tilde{\eta}_{0}(\tilde{t}) \quad \text { and } \quad \tilde{\eta}(\tilde{t}, L)=\tilde{\eta}_{L}(\tilde{t})
\end{gathered}
$$

where $\beta_{0}>0$ is a given constant. We apply the Laplace transform to (A.4), (A.5) and obtain

$$
\begin{gathered}
S h_{0} p \hat{\tilde{\eta}}(p, \tilde{z})-\beta_{0} R e_{0} S h_{0} \hat{\tilde{\mathcal{K}}}\left(R e_{0} S h_{0} p\right) \frac{\partial^{2}}{\partial \tilde{z}^{2}} \hat{\tilde{\eta}}(p, \tilde{z})=0 \\
\hat{\tilde{\eta}}(p, 0)=\hat{\tilde{\eta}}_{0}(p) \quad \text { and } \quad \hat{\tilde{\eta}}(p, L)=\hat{\tilde{\eta}}_{L}(p)
\end{gathered}
$$


Let

$$
\beta(p):=S h_{0} \frac{p^{2}}{\beta_{0}}\left\{1-\frac{2}{\sqrt{R e_{0} S h_{0} p}} \frac{I_{1}\left(\sqrt{R e_{0} S h_{0} p}\right)}{I_{0}\left(\sqrt{R e_{0} S h_{0} p}\right)}\right\}^{-1} .
$$

Then the solution of (A.4), (A.5) is given by

$$
\hat{\tilde{\eta}}(p, \tilde{z})=\frac{\hat{\tilde{\eta}}_{L}(p)-\hat{\tilde{\eta}}_{0}(p) \cosh (\sqrt{\beta(p)} L)}{\sinh (\sqrt{\beta(p)} L)} \sinh (\sqrt{\beta(p)} \tilde{z})+\hat{\tilde{\eta}}_{0}(p) \cosh (\sqrt{\beta(p)} \tilde{z}) .
$$

Appendix B. Existence, regularity and uniqueness for the Biot System. We prove here that the system studied in $\S 7.2 .4$ has a unique solution. Consider

$$
\begin{gathered}
\frac{\partial \eta}{\partial t}+\gamma_{1} \frac{\partial}{\partial z} \int_{0}^{1} r v_{z} d r=-\frac{\partial \eta_{L}}{\partial t} \quad \text { in } \quad(0, L) \times(0, T), \\
\frac{\partial v_{z}}{\partial t}-\frac{1}{r} \frac{\partial}{\partial r}\left(r \frac{\partial v_{z}}{\partial r}\right)=-\gamma_{2} \frac{\partial \eta}{\partial z}-\gamma_{2} \frac{\partial \eta_{L}}{\partial z} \quad \text { in } \quad(0,1) \times(0, L) \times(0, T), \\
v_{z}(1, z, t)=0, \quad v_{z}(0, z, t) \quad \text { bounded, } \quad \eta(0, t)=\eta(L, t)=0 \\
\eta(z, 0)=v_{z}(r, z, 0)=0 \quad \text { on } \quad(0,1) \times(0, L),
\end{gathered}
$$

where

$$
\eta_{L}(z, t)=\frac{\eta_{L}(t)-\eta_{0}(t)}{L} z+\eta_{0}(t)
$$

and $\eta_{0}, \eta_{L} \in C_{0}^{\infty}(0, \infty), \gamma_{1}$ and $\gamma_{2}$ are positive constants. System (B.1)-(B.4) implies the following energy equalities

$$
\begin{gathered}
\frac{1}{2} \frac{d}{d t}\left\{\int_{0}^{L}|\eta(z, t)|^{2} d z+\frac{\gamma_{1}}{\gamma_{2}} \int_{0}^{L} \int_{0}^{1}\left|v_{z}(r, z, t)\right|^{2} r d r d z\right\} \\
+\frac{\gamma_{1}}{\gamma_{2}} \int_{0}^{L} \int_{0}^{1}\left|\frac{\partial v_{z}}{\partial r}\right|^{2} r d r d z=\int_{0}^{L} \frac{\partial \eta_{L}}{\partial t} \eta d z-\gamma_{1} \int_{0}^{L} \int_{0}^{1} r v_{z} \frac{\partial \eta_{L}}{\partial z} d r d z \\
\frac{1}{2} \frac{d}{d t}\left\{\int_{0}^{L}\left|\frac{\partial \eta}{\partial t}\right|^{2} d z+\frac{\gamma_{1}}{\gamma_{2}} \int_{0}^{L} \int_{0}^{1}\left|\frac{\partial v_{z}}{\partial t}\right|^{2} r d r d z\right\} \\
+\frac{\gamma_{1}}{\gamma_{2}} \int_{0}^{L} \int_{0}^{1}\left|\frac{\partial^{2} v_{z}}{\partial t \partial r}\right|^{2} r d r d z=\int_{0}^{L} \frac{\partial^{2} \eta_{L}}{\partial t^{2}} \frac{\partial \eta}{\partial t} d z-\gamma_{1} \int_{0}^{L} \int_{0}^{1} r \frac{\partial v_{z}}{\partial t} \frac{\partial \eta_{L}}{\partial z} d r d z .
\end{gathered}
$$

Since (B.6) guarantees that $\frac{\partial v_{z}}{\partial t} \in L^{2}$, we write (B.2) in the form

$$
-\Delta_{r} v_{z}:=-\frac{1}{r} \frac{\partial}{\partial r}\left(r \frac{\partial v_{z}}{\partial r}\right)=-\gamma_{2} \frac{\partial \eta}{\partial z}(z, t)-\gamma_{2} \frac{\partial \eta_{L}}{\partial z}-\frac{\partial v_{z}}{\partial t}
$$

and, consequently

$$
\int_{0}^{1} r v_{z} d r=-\frac{\gamma_{2}}{16} \frac{\partial \eta}{\partial z}(z, t)-\frac{\gamma_{2}}{16} \frac{\partial \eta_{L}}{\partial z}-\int_{0}^{1} r\left(-\Delta_{r}\right)^{-1} \frac{\partial v_{z}}{\partial t} d r
$$

Using this expression in equation (B.1) we get

$$
\frac{\partial \eta}{\partial t}-\frac{\gamma_{1} \gamma_{2}}{16} \frac{\partial^{2} \eta}{\partial z^{2}}=-\frac{\partial \eta_{L}}{\partial t}+\frac{\gamma_{1} \gamma_{2}}{16} \frac{\partial^{2} \eta_{L}}{\partial z^{2}}+\gamma_{1} \frac{\partial}{\partial z} \int_{0}^{1} r\left(-\Delta_{r}\right)^{-1} \frac{\partial v_{z}}{\partial t} d r .
$$


We multiply this equation by $\eta$ and integrate. After taking into account that $\frac{\partial^{2} \eta_{L}}{\partial z^{2}}=0$ we obtain a standard energy estimate for the heat equation

$$
\begin{gathered}
\frac{1}{2} \int_{0}^{L}|\eta(z, t)|^{2} d z+\frac{\gamma_{1} \gamma_{2}}{16} \int_{0}^{t} \int_{0}^{L}\left|\frac{\partial \eta}{\partial z}\right|^{2} d z d \tau=-\int_{0}^{t} \int_{0}^{L} \frac{\partial \eta_{L}}{\partial t} \eta d z d \tau \\
-\gamma_{1} \int_{0}^{t} \int_{0}^{L}\left(\int_{0}^{1} r\left(-\Delta_{r}\right)^{-1} \frac{\partial v_{z}}{\partial t} d r\right) \frac{\partial \eta}{\partial z} d z d \tau .
\end{gathered}
$$

The a priori estimates (B.5), (B.6) and (B.7) imply existence of a unique solution $\left\{\eta, v_{z}\right\} \in H^{1}((0, L) \times(0, T)) \times H^{1}\left(0, T ; L^{2}((0,1) \times(0, L))\right), \sqrt{r} \frac{\partial v_{z}}{\partial r} \in H^{1}\left(0, T ; L^{2}((0,1) \times\right.$ $(0, L))$ for the Biot system (B.1)-(B.4). This regularity guarantees uniqueness of a solution to system (7.2)-(7.6). We have proved the following

Theorem B.1. The Biot system (B.1)-(B.4) has a unique solution $\left\{\eta, v_{z}\right\} \in$ $H^{1}((0, L) \times(0, T)) \times H^{1}\left(0, T ; L^{2}((0,1) \times(0, L))\right)$ with $\sqrt{r} \frac{\partial v_{z}}{\partial r} \in H^{1}\left(0, T ; L^{2}((0,1) \times\right.$ $(0, L))$.

Corollary B.2. A solution of system (7.2)-(7.6) is unique.

Acknowledgments. The authors would like to thank Prof. Alfio Quarteroni for his support, encouragement and stimulating discussions. Also, the authors would like to thank the referees for their careful reading of the manuscript and for many useful suggestions. Support by the Texas Higher Education Board ARP(Mathematics) under grant number 003652-0112-2001 was particularly beneficial to the project. The National Science Foundation support under grants DMS9970310 and DMS0245513 is also acknowledged.

\section{REFERENCES}

[1] M. A. Biот, Theory of propagation of elastic waves $n$ a fluid-saturated porous solid. I. Lower frequency range, and II. Higher frequency range, J. Acoust. Soc. Am., 28 (2) (1956), pp. $168-178$ and pp. $179-191$.

[2] Y. Brenier, Homogeneous hydrostatic flows with convex velocity profiles. Nonlinearity, 12 (1999), pp. 495-512.

[3] S. ČANIĆ, Blood flow through compliant vessels after endovascular repair: wall deformations induced by the discontinuous wall properties. Comp. Visual. Sci., 4(3) (2002), pp. 147155.

[4] S. ČANIĆ AND E-H. KIM, Mathematical analysis of the quasilinear effects in a hyperbolic model of blood flow through compliant axi-symmetric vessels, Mathematical Methods in the Applied Sciences, 26(14) (2003), pp. 1161-1186.

[5] S. ČANIĆ AND A. Mikelić, Effective equations describing the flow of a viscous incompressible fluid through a long elastic tube, Comptes Rendus Mécanique Acad. Sci. Paris, 330 (2002), pp. 661-666.

[6] S. ČANIĆ AND A. Mikelić, Effective equations modeling the flow of a viscous incompressible fluid through a long elastic tube arising in the study of blood flow through small arteries., SIAM Journal on Applied Dynamical Systems, 2(3) (2003), pp. 431-463.

[7] S. ČAnić And A. Mikelić and J. TAmbaČA, Coupling between the flow of a viscous incompressible fluid and the nolinearly elastic cylindrical shell of Koiter type: the effective equations and a numerical solver. In draft form.

[8] P. G. Ciarlet, Plates and junctions in elastic multi-structures, R.M.A. 14, Masson, Paris, 1990.

[9] C. Chmielevsky, "A Comparison of Two Models Predicting Blood Flow in the Systemic Arteries, M.S.Thesis, North Carolina State University (2004).

[10] L. Formaggia, F. Nobile, And A. Quarteroni, A one dimensional model for blood flow: application to vascular prosthesis, in Mathematical Modeling and Numerical Simulation in Continuum Mechanics, I. Babuska, T. Miyoshi and P. G. Ciarlet, eds., Lecture Notes in Computational Science and Engineering, 19 (2002), pp. 137-153.

[11] Y.C. Fung, Biomechanics: Circulation, Springer, New York, 1993. Second Edition. 
[12] Y.C. Fung, Biomechanics: Mechanical Properties of Living Tissues, Springer, New York, 1993.

[13] R. Glowinski, T. W. Pan and J. Periaux, A fictitious domain method for Dirichlet problem and applications. Comp. Meth. Appl. Mech. Engrg. 111(3-4) (1994), pp. 283-303.

[14] R. Glowinski Finite Element Methods for Incompressible Viscous Flow, Vol IX of Handbook of Numerical Analysis, P. G. Ciarlet and J. L. Lions, eds., North-Holland, Amsterdam, 2003.

[15] A. Chambolle, B. Desjardins, M.-J. Esteban and C. Grandmont, Existence of weak solutions for an unsteady fluid-plate interaction problem, preprint.

[16] J. Keener and J. Sneyd Mathematical Physiology, Interdisciplinary Applied Mathematics. Vol. 8, Springer, New York, 1998.

[17] R.E. KLABunde, Cardiovascular Physiology Concepts, Web-based resource http://cvphysiology.com/index.html.

[18] K. Kröger, K. Massalha, C. Buss and G. Rudofsky, Effect of hemodynamic conditions on sonographic measurements of peak systolic velocity and arterial diameter in patients with peripheral arterial stenosis, Journal of Clinical Ultrasound bf 28(3) (2000), pp. 109-114.

[19] P. Luchini, M. Lupo And A. Pozzi, Unsteady Stokes flow in a distensible pipe, Z. angew. Math. Mech. 71 (1991), pp. 367-378.

[20] X. MA, G.C. Lee ANd S.G. Lu, Numerical Simulation for the ropagation of Nonlinear Pulsatile Waves in Arteries, ASME J. Biomech. Eng. 114 (1992), pp. 490-496.

[21] S.G. Wu, G.C. Lee And N.T. Tseng, Nonlinear Elastic Analysis of Blood Vessels, J. Biomech. Engrg. 106 (1984), pp. 376-383.

[22] E. Marušić-PALOKA AND A. Mikelić, The derivation of a nonlinear filtration law including the inertia effects via homogenization, Nonlinear Analysis 42 (2000), pp. 97-137.

[23] W. W. Nichols and M. F. O'Rourke, McDonald's Blood Flow in Arteries: Theoretical, experimental and clinical principles, Fourth Edition, Arnold and Oxford University Press Inc., New York, London, Sydney, Auckland, 1997.

[24] A. Mikelić, Homogenization theory and applications to filtration through porous media, in Filtration in Porous Media and Industrial Applications, by M. Espedal, A. Fasano and A. Mikelić, Lecture Notes in Mathematics Vol. 1734, Springer, 2000, pp. 127-214.

[25] R. Murata, H. Kanai, N. Chubachi and Y. Koiwa, Measurement of Local Pulse Wave Velocity on Aorta for Noninvasive Diagnosis of Arteriosclerosis, IEEE Engineering in Medicine and Biology (1994), paper 521.

[26] F. NoBlLe, Numerical Approximation of Fluid-Structure Interaction Problems with Application to Haemodynamics, Ph.D. Thesis, EPFL, Lausanne, 2001.

[27] M.S. Olufsen, A. NAdim, And L.A. Lipsitz, Dynamics of cerebral blood flow regulation explained using a lumped parameter model, American Journal of Physiology Integrative Computational Physiology, 282:R611-R622, 2002.

[28] M. S. Olufsen, C. S. Peskin, W. Y. Kim, E. M. Pedersen, A. Nadim and J. Larsen, Numerical Simulation and Experimental Validation of Blood Flow in Arteries with Structured-Tree Outflow Conditions, Annals of Biomedical Engineering 28 (2000), pp. 1281-1299.

[29] T. J. PedLey, The Fluid Mechanics of Large Blood Vessels, Cambridge University Press, Cambridge, U.K., 1980.

[30] K. Perktold, And G. Rappitsch, Mathematical modeling of local arterial flow and vessel mechanics, in Computational Methods for Fluid Structure Interaction, J. Crolet and R. Ohayon, eds., Pitman Research Notes in Mathematics 306, Harlow, Longman, 1994, pp. 230-245.

[31] C.S. Peskin And D.M. McQueen, A Three-Dimensional Computational Method for Blood Flow in the Heart - I Immersed Elastic Fibers in a Viscous Incompressible Fluid, J. Comp. Phys. 81(2) (1989), pp. 372-405.

[32] H. Reismann, Elastic Plates: Theory and Applications, Wiley, New York, 1988.

[33] A.M. Robertson And A Sequeira, A director theory approach to modeling blood flow in the arterial system, submitted.

[34] N. P. Smith, A. J. Pullan and P. J. Hunter, An Anatomically Based Model of Transient Coronary Blood Flow in the Heart. SIAM J. Appl. Math. 62(3) (2002), pp. 990-1018.

[35] L. TARTAR, Mathematical tools for studying oscillations and concentrations: from Young measures to $H$-measures and their variants, in Multiscale Problems in Science and Technology: Challenges to Mathematical Analysis and Perspectives, (Dubrovnik, 2000), N. Antonić et al., eds., Springer-Verlag, Heidelberg, 2002, pp. 1-84.

[36] C. A. Taylor, T. J. R. Hughes and C. K. Zarins, Effect of Exercise on Hemodynamic Conditions in the Abdominal Aorta. Journal of Vascular Surgery, 29(6) (1999), pp. 1077- 
1089.

[37] R. Temam, Navier-Stokes Equations, North-Holland, 1984.

[38] A. Quarteroni, M. Tuveri and A. Veneziani, Computational vascular fluid dynamics: problems, models and methods. Survey article, Comput. Visual. Sci. 2 (2000), pp. 163197.

[39] J. R. Womersley, Method for the calculation of velocity, rate of flow and viscous drag in arteries when the pressure gradient is known, J. Physiol. 127 (1955), pp. 553-563. 